\title{
Scalar mesons moving in a finite volume and the role of partial wave mixing
}

\author{
M. Döring ${ }^{1}$, U.-G. Meißner ${ }^{1,2}$, E. Oset ${ }^{3}$ and A. Rusetsky ${ }^{1}$ \\ ${ }^{1}$ Helmholtz-Institut für Strahlen- und Kernphysik (Theorie) and Bethe Center for Theoretical Physics, \\ Universität Bonn, Nußallee 14-16, D-53115 Bonn, Germany \\ ${ }^{2}$ Forschungszentrum Jülich, Jülich Center for Hadron Physics, \\ Institut für Kernphysik (IKP-3) and Institute for Advanced Simulation (IAS-4), D-52425 Jülich, Germany \\ ${ }^{3}$ Departamento de Física Teórica and IFIC, Centro Mixto Universidad de Valencia-CSIC, \\ Institutos de Investigación de Paterna, Aptdo. 22085, 46071 Valencia, Spain
}

\begin{abstract}
Phase shifts and resonance parameters can be obtained from finite-volume lattice spectra for interacting pairs of particles, moving with nonzero total momentum. We present a simple derivation of the method that is subsequently applied to obtain the $\pi \pi$ and $\pi K$ phase shifts in the sectors with total isospin $I=0$ and $I=1 / 2$, respectively. Considering different total momenta, one obtains extra data points for a given volume that allow for a very efficient extraction of the resonance parameters in the infinite-volume limit. Corrections due to the mixing of partial waves are provided. We expect that our results will help to optimize the strategies in lattice simulations, which aim at an accurate determination of the scattering and resonance properties.

PACS numbers: 11.80.Gw, 12.38.Gc, 12.39.Fe, 13.75.Lb
\end{abstract}

\section{INTRODUCTION}

One of the present issues in QCD lattice calculations is the determination of the excited hadron spectrum. Many efforts are being devoted to this problem lately [1 27]. In the volume-dependent spectrum, the "avoided level crossing" is usually taken as a signal of a resonance, but this criterion has been shown insufficient for resonances with a large width 28,32$]$. For resonances with a single decay channel, one often uses Lüscher's approach to extract phase shifts from the discrete energy levels in the box [33, 34]. The method has been recently extended to multi-channel scattering [31, 35-37] and to the case with three-particle intermediate states [38, 39]. Moreover, in Ref. [32], a method based on coupled-channel Unitary Chiral Perturbation Theory (UChPT), which operates with the full relativistic two-body propagator, has been proposed. In the infinite-volume limit, this method is equivalent to Lüscher's approach, up to contributions (kept in Ref. [32]), which are exponentially suppressed in this limit. The new method, combining conceptual and technical simplicity, can provide a guideline for future lattice calculations.

This method has been extended in Ref. [40] for the use in connection with dynamical coupled-channel approaches such as the Jülich model [41 43]. In Ref. [44] the framework of Ref. 32] has been applied for the interaction of the $D K$ and $\eta D_{s}$ system, where the $D_{s^{*} 0}(2317)$ resonance is dynamically generated from the interaction of these particles. The case of the $\kappa$ in the $K \pi, K \eta$ channels is addressed along the lines of Ref. [32] in Ref. [45], together with the $\sigma(600), K^{*}(892)$, and $\rho$ resonances. The formalism has also been extended to the case of the interaction of unstable particles in Ref. [46], to the study of the $D N$ interaction [47], and the $\pi \pi$ interaction in the $\rho$ channel [48]. Pioneering work for the coupled-channel $\bar{K} N, \pi \Sigma$ system and the $\Lambda(1405)$ in the finite volume has been carried out in Ref. [37]; the lattice levels for the
$\Lambda(1405)$ quantum numbers were evaluated in Ref. [40] using the Jülich model, and strategies to determine the two $\Lambda(1405)$ states from lattice results (c.f. also Ref. 23]) were discussed in Ref. [49]. Methods to extract matrix elements of unstable particles from the finite volume have been recently developed in Ref. 50].

The derivation of Refs. [33, 34] or Ref. 32] is done for a pair of particles with total zero-momentum. The generalization to a moving frame has been done in 39, 51 60.

In this study we present an easy derivation of the approach for moving frames, along the lines of Ref. [32], using fully relativistic propagators and arbitrary masses, and we apply the method to study the coupled-channel scattering of $\pi \pi$ and $\pi K$ in the region of the $f_{0}(600)$ and $\kappa(800)$ resonances, respectively.

The strategy followed here is to use the chiral unitary approach to generate synthetic lattice data, which are later on analyzed to extract phase shifts and resonance properties. We show that the data produced with moving frames are very useful to get the infinite-volume properties using two box volumes and different total momenta. The study done here permits to find optimal strategies, concerning which lattice data to use, in order to obtain phase shifts and resonance properties with maximum precision.

\section{FORMALISM}

\section{A. Particles in a moving frame}

In the chiral unitary approach the scattering matrix in coupled channels is given by the Bethe-Salpeter equation (BSE) in its factorized form

$$
T=[1-V G]^{-1} V=\left[V^{-1}-G\right]^{-1},
$$


where $V \equiv V^{(i j)}$ is the matrix for the transition potentials between the channels and $G$ is a diagonal matrix with the $i^{\text {th }}$ element, $G^{(i)}$, given by the loop function of two propagators, which for two mesons is defined as

$$
G^{(i)}=i \int \frac{d^{4} q}{(2 \pi)^{4}} \frac{1}{(P-q)^{2}-m_{1}^{2}+i \epsilon} \frac{1}{q^{2}-m_{2}^{2}+i \epsilon},
$$

where $m_{i}$ are the masses of the two mesons and $P$ the total four-momentum of the meson-meson system. The factorized form of the BSE implies an on-shell factorization of the potential $V$, see Sec. IIC for a discussion.

$V$ and $T$ in Eq. (10) stand for the potential and scattering matrix in the momentum space. The normalization is such that, for the case of one channel, we have

$$
\begin{aligned}
& \frac{1}{2 i}\left(e^{2 i \delta}-1\right)=e^{i \delta} \sin \delta=\frac{1}{\cot \delta-i} \\
& =-\frac{p}{8 \pi E} T=-\frac{p}{8 \pi E} \frac{V}{1-V G}=\frac{1}{2 i}(S-1),
\end{aligned}
$$

which relates $T$ to the phase shift $\delta$ and the $S$-matrix, where $E$ is the total energy in the CM and $p$ the momentum, $p=\lambda^{1 / 2}\left(E^{2}, m_{1}^{2}, m_{2}^{2}\right) /(2 E)$. For the twochannel case, corresponding relations can be found in Refs. 62, 63].

The loop function in Eq. (2) needs to be regularized and this can be accomplished either with dimensional regularization or with a three-momentum cutoff. The equivalence of both methods was shown in Refs. [61, 62. In the regularization with a three-momentum cutoff, one first performs the $q^{0}$ integration analytically [63]. As a result, one gets

$$
G^{(i)}=\int^{|\vec{q}|<q_{\max }} \frac{d^{3} \vec{q}}{(2 \pi)^{3}} I^{(i)}(|\vec{q}|)
$$

where

$$
\begin{aligned}
I^{(i)}(|\vec{q}|) & =\frac{1}{2 \omega_{1}(\vec{q}) \omega_{2}(\vec{q})} \frac{\omega_{1}(\vec{q})+\omega_{2}(\vec{q})}{E^{2}-\left(\omega_{1}(\vec{q})+\omega_{2}(\vec{q})\right)^{2}+i \epsilon} \\
\omega_{j}^{2} & =m_{j}^{2}+\vec{q}^{2}
\end{aligned}
$$

We would like to stress that other renormalization schemes such as dimensional regularization are, in general, preferable over the cut-off renormalization. However, note that for the extraction of the infinite-volume limit from lattice levels the cut-off dependence cancels as discussed in Sec. II G The treatment of finite volume using dimensional regularization is done in Ref. [44].

To obtain the energy levels in the finite box, instead of integrating over the momenta of the continuum - with $q$ being a continuous variable as in Eq. (4) - one must sum over the discrete momenta allowed in a finite box of side length $L$ with periodic boundary conditions. We then have to replace $G$ by $\tilde{G}=\operatorname{diag}\left(\tilde{G}^{(1)}, \tilde{G}^{(2)}\right)$, where

$$
\begin{aligned}
\tilde{G}^{(i)} & =\frac{1}{L^{3}} \sum_{\vec{n}}^{|\vec{q}|<q_{\max }} I^{(i)}(|\vec{q}|), \\
\vec{q} & =\frac{2 \pi}{L} \vec{n}, \quad \vec{n} \in \mathbb{Z}^{3} .
\end{aligned}
$$

This is the procedure followed in 32]. Here and in the following, we indicate quantities in the finite volume with a tilde, e.g. $G \rightarrow \tilde{G}$, or $T \rightarrow \tilde{T}$.

The eigenenergies of the box correspond to energies that produce poles in the $\tilde{T}$ scattering matrix in the finite volume,

$$
\tilde{T}=[1-V \tilde{G}]^{-1} V=\left[V^{-1}-\tilde{G}\right]^{-1}
$$

i.e. for energies where $\operatorname{det}(1-V \tilde{G})=0$.

In the former discussion the integrals and sums are performed in the rest frame of the two interacting particles. Yet, since in the infinite volume the $G$ function is Lorentz invariant, see Eq. (2), it suffices to evaluate it in the two-particle rest frame. In another frame it will take the same value as required by Lorentz invariance. Let us call $q^{*} \equiv\left|\vec{q}^{*}\right|$ the absolute value of the relative threemomentum in the rest frame of the two particles, called center-of-mass frame (CM) in the following. The absolute value of the relative three-momentum in a frame where the two-particle system has total momentum $\left(P^{0}, \vec{P}\right)$ is called $q$. The CM energy of the two-particle system will be $\sqrt{s}$, such that

$$
s \equiv E^{2}=\left(P^{0}\right)^{2}-\vec{P}^{2} .
$$

However, for the system moving in the finite volume, Lorentz invariance is broken and hence we cannot use $\tilde{G}$ evaluated in the CM, because the discretization condition in the momenta $\overrightarrow{q_{1}}$ and $\overrightarrow{q_{2}}=\vec{P}-\overrightarrow{q_{1}}$ of the particles of Eq. (6) must be transformed to the moving frame. We must write the boost transformation from $q$ to $q^{*}$. By applying the Lorentz transformation from a moving frame with four-momentum $P$ to a frame where the two particle system is at rest we find

$$
\vec{q}_{1,2}^{*}=\vec{q}_{1,2}+\left[\left(\frac{P^{0}}{\sqrt{s}}-1\right) \frac{\vec{q}_{1,2} \cdot \vec{P}}{|\vec{P}|^{2}}-\frac{q_{1,2}^{0}}{\sqrt{s}}\right] \vec{P}
$$

Here and in the following, a star indicates a quantity defined in the two-particle rest frame.

Demanding that $\overrightarrow{q_{1}^{*}}+\overrightarrow{q_{2}^{*}}=0$ enforces $q_{1}^{0}+q_{2}^{0}=P^{0}$. We also have the transformation of the energies

$$
q_{1,2}^{0}=\frac{\left(q_{1,2}^{* 0} \sqrt{s}+\vec{q}_{1,2} \cdot \vec{P}\right)}{P^{0}}
$$

and the condition $q_{1}^{0}+q_{2}^{0}=P^{0}$ imposes

$$
q_{1}^{* 0}+q_{2}^{* 0}=\sqrt{s}
$$


which on shell gives

$$
q_{1,2}^{* 0}=\frac{s+m_{1,2}^{2}-m_{2,1}^{2}}{2 \sqrt{s}} .
$$

This, via Eqs. (9) and (10), provides then the boost for the off-shell momenta in the loop, where $\vec{q}$ is arbitrary but the energy is the on-shell one. Only this prescription ensures $q_{1}^{* 0}=q_{2}^{* 0}$ for two particles of equal mass in the two-particle rest frame. Since we need the Jacobian of this transformation, it is useful to rewrite Eq. (9) in terms of the CM energy of the particles and we find

$$
\vec{q}_{1,2}^{*}=\vec{q}_{1,2}+\left[\left(\frac{\sqrt{s}}{P^{0}}-1\right) \frac{\vec{q}_{1,2} \cdot \vec{P}}{|\vec{P}|^{2}}-\frac{q_{1,2}^{* 0}}{P^{0}}\right] \vec{P}
$$

This equation is the one used in 64. Furthermore we must substitute $\int d^{3} \vec{q}^{*} /(2 \pi)^{3}$ by $\int d^{3} \vec{q} /(2 \pi)^{3} \sqrt{s} / P^{0}$, where the factor $\sqrt{s} / P^{0}$ is the Jacobian of the transformation, and then replace the integral by the discrete sum. In summary, we must perform the substitution

$$
\int \frac{d^{3} \vec{q}^{*}}{(2 \pi)^{3}} I\left(\left|\vec{q}^{*}\right|\right) \longrightarrow \tilde{G}(P)=\frac{1}{L^{3}} \frac{\sqrt{s}}{P^{0}} \sum_{\vec{n}} I\left(\left|\vec{q}^{*}(\vec{q})\right|\right)
$$

with

$$
\vec{q}=\frac{2 \pi}{L} \vec{n}, \quad \vec{n} \in \mathbb{Z}^{3},
$$

where for both the sum and the integral the limit is $\left|\vec{q}^{*}\right|<$ $q_{\max }$.

Note that in order to have both $\overrightarrow{q_{1}}$ and $\overrightarrow{q_{2}}=\vec{P}-\overrightarrow{q_{1}}$ fulfilling the periodic boundary conditions, the momentum $\vec{P}$ must fulfill them, too, and thus we have

$$
\vec{P}=\frac{2 \pi}{L} \vec{N}, \quad \vec{N} \in \mathbb{Z}^{3} .
$$

A clarification on the role of the on-shell reduction used in Eq. (1) is appropriate. In general, one does not know how good this approximation is. However, in the infinitevolume limit, the on-shell approximation of the potential $V$ derived from a Lagrangian delivers in many cases a successful description of the phenomenology, see, e.g., the hadronic model we use in this work [62]. However, fully covariant off-shell unitarized approaches have been also developed 65 67]. Off-shell unitarized approaches in a three-dimensional reduction are realized, e.g., in dynamical coupled-channel models [41 43].

While there are a conceptual differences between onand off-shell approaches in the infinite volume limit, it should be clearly stated that, whatever approach is chosen in the infinite volume limit, the levels in the finite volume are determined by the on-shell amplitude up to exponentially suppressed effects $\sim e^{-L M_{\pi}}$.

\section{B. One-channel analysis}

The one-channel problem can be easily solved and is very simple, as shown in 32. The $T$ matrix for the infinite volume can be obtained for the energies which are eigenvalues of the box by $(E=\sqrt{s})$

$$
T(E)=\left(V^{-1}(E)-G(E)\right)^{-1}=(\tilde{G}(P)-G(E))^{-1} .
$$

since $\tilde{G}(P)=V^{-1}(E)$ is the condition for the $\tilde{T}$ matrix to have a pole for the finite box.

Hence we find, in the one-channel case and assuming that only $S$-wave scattering is present,

$$
\begin{aligned}
T(E)^{-1}=\lim _{q_{\max } \rightarrow \infty} & {\left[\frac{1}{L^{3}} \sum_{\vec{n}}^{\left|\vec{q}^{*}\right|<q_{\max }} \frac{E}{P^{0}} I\left(\left|\vec{q}^{*}(\vec{q})\right|\right)\right.} \\
& \left.-\int_{\left|\vec{q}^{*}\right|<q_{\max }} \frac{d^{3} \vec{q}^{*}}{(2 \pi)^{3}} I\left(\left|\vec{q}^{*}\right|\right)\right] .
\end{aligned}
$$

This derivation is very simple and the results can be seen to agree with previous ones [51, 52, 55, 56] when one approximates $I\left(q^{*}\right)$ by

$$
I\left(q^{*}\right) \rightarrow \frac{1}{2 E} \frac{1}{p^{2}-\left(\vec{q}^{*}\right)^{2}+i \epsilon}
$$

where $p=\lambda^{1 / 2}\left(E^{2}, m_{1}^{2}, m_{2}^{2}\right) /(2 E)$ and $\lambda(x, y, z)$ stands for the Källén triangle function. It is seen that, with this replacement, our expressions agree with those in the Lüscher framework. Furthermore, summing the difference of Eq. (5) and Eq. (18) over the momenta, it is immediately seen that the finite-volume corrections to this quantity are exponentially suppressed. Consequently, the present approach is equivalent to Lüscher's approach up to the exponentially suppressed terms for large volumes. Note however that, for moderately large volumes, these exponentially suppressed terms can be important numerically, see the discussion in Ref. [32]. One should realize, however, that for values of $L M_{\pi}$ where these terms play a role, there are many other exponentially suppressed corrections.

We would like to stress that the formal dependence on the potential $V$ cancels in Eq. (17) - in other words, Eq. (17) contains $T$ and does not contain $V$ or $G$ individually. This was expected from the beginning, because the potential $V$ is not an observable and depends on the cut-off chosen for $G$. For the case of moving frames and partial wave mixing, discussed in the following, we will find exactly the same behavior: the measured lattice levels depend only on the $T$-matrix in the infinite volume and and functions $\tilde{G}-G$, which are cutoff-independent.

\section{Partial wave decomposition in a finite volume}

To determine the mixing of partial waves, consider first the case without boost, i.e. $\vec{P}=\overrightarrow{0}$. At the end of this section, the formalism is generalized to moving frames and multiple channels. 
We use the spherical harmonics $Y_{\ell m}$ with the normalization

$$
\int_{0}^{2 \pi} d \phi \int_{0}^{\pi} \sin \theta d \theta Y_{\ell m}(\theta, \phi) Y_{\ell^{\prime} m^{\prime}}^{*}(\theta, \phi)=\delta_{\ell \ell^{\prime}} \delta_{m m^{\prime}}
$$

and further define

$$
\begin{aligned}
\mathcal{Y}_{\ell m}(\vec{p}) & =p^{\ell} Y_{\ell m}(\theta, \phi)=p^{\ell} Y_{\ell m}(\hat{p}), \\
\vec{p} & =p(\sin \theta \cos \phi, \sin \theta \sin \phi, \cos \theta)=p \hat{p} .
\end{aligned}
$$

The potential $V$ in the Bethe-Salpeter equation (1) is the same in a finite and in the infinite volume, $V=\tilde{V}$. Its partial-wave expansion takes the standard form

$$
V\left(\vec{p}, \vec{p}^{\prime}\right)=4 \pi \sum_{\ell, m} \mathcal{Y}_{\ell m}(\vec{p}) v_{\ell}\left(p, p^{\prime}\right) \mathcal{Y}_{\ell m}^{*}\left(\vec{p}^{\prime}\right)
$$

However, the partial-wave expansion of the scattering amplitude $\tilde{T}$ is different in a finite volume, because here the rotational symmetry is broken down to cubic symmetry. As a result,

$$
\tilde{T}\left(\vec{p}, \vec{p}^{\prime}\right)=4 \pi \sum_{\substack{\ell, m \\ \ell^{\prime} m^{\prime}}} \mathcal{Y}_{\ell m}(\vec{p}) t_{\ell m, \ell^{\prime} m^{\prime}}\left(p, p^{\prime}\right) \mathcal{Y}_{\ell^{\prime} m^{\prime}}^{*}\left(\vec{p}^{\prime}\right)
$$

In the above expressions, $v_{\ell}$ and $t_{\ell m, \ell^{\prime} m^{\prime}}$ depend only on $p^{2}=\vec{p}^{2}$ and $\left(p^{\prime}\right)^{2}=\left(\vec{p}^{\prime}\right)^{2}$. Note that the threshold behavior of the amplitude is hidden in the function $\mathcal{Y}_{\ell m}(\vec{p}) \sim p^{\ell}$.

In the infinite-volume limit, the rotational symmetry is restored and the Wigner-Eckart theorem guarantees that $t$ is diagonal both in $\ell$ and $m$,

$$
t_{\ell m, \ell^{\prime} m^{\prime}}=t_{\ell} \delta_{\ell \ell^{\prime}} \delta_{m m^{\prime}}
$$

In contrast, in the finite volume one obtains

$$
\begin{aligned}
& t_{\ell m, \ell^{\prime} m^{\prime}}\left(p, p^{\prime}\right)=v_{\ell}\left(p, p^{\prime}\right) \delta_{\ell \ell^{\prime}} \delta_{m m^{\prime}}+\frac{4 \pi}{L^{3}} \sum_{\vec{n}} \sum_{\ell^{\prime \prime} m^{\prime \prime}} \\
& \times v_{\ell}(p, q) \mathcal{Y}_{\ell m}^{*}(\vec{q}) I(q) \mathcal{Y}_{\ell^{\prime \prime} m^{\prime \prime}}(\vec{q}) t_{\ell^{\prime \prime} m^{\prime \prime}, \ell^{\prime} m^{\prime}}\left(q, p^{\prime}\right)
\end{aligned}
$$

with $I(q)$ from Eq. (5). This relation is obtained straightforwardly by inserting $V$ from Eq. (21) and $\tilde{T}$ from Eq. (22) in Eq. (7).

Next, note that, since $v_{\ell}\left(p, p^{\prime}\right)$ and $t_{\ell m, \ell^{\prime} m^{\prime}}\left(p, p^{\prime}\right)$ depend only on $p^{2},\left(p^{\prime}\right)^{2}$, the use of the regular summation theorem [33] is justified. Further, the argument $q$ in these functions [see Eq. (24)] can be replaced by the on-shell value $q^{\text {on }}$, which is determined from the zero of the energy denominator in Eq. (5). Finally, using Eq. (20), one gets

$$
\begin{aligned}
& \tilde{T}_{\ell m, \ell^{\prime} m^{\prime}}\left(p, p^{\prime}\right)=V_{\ell}\left(p, p^{\prime}\right) \delta_{\ell \ell^{\prime}} \delta_{m m^{\prime}} \\
& +\sum_{\ell^{\prime \prime} m^{\prime \prime}} V_{\ell}\left(p, q^{\mathrm{on}}\right) \tilde{G}_{\ell m, \ell^{\prime \prime} m^{\prime \prime}}\left(q^{\mathrm{on}}\right) \tilde{T}_{\ell^{\prime \prime} m^{\prime \prime}, \ell^{\prime} m^{\prime}}\left(q^{\mathrm{on}}, p^{\prime}\right),
\end{aligned}
$$

where

$$
\begin{aligned}
\tilde{T}_{\ell m, \ell^{\prime} m^{\prime}}\left(p, p^{\prime}\right) & =p^{\ell} t_{\ell m, \ell^{\prime} m^{\prime}}\left(p, p^{\prime}\right)\left(p^{\prime}\right)^{\ell^{\prime}} \\
V_{\ell}\left(p, p^{\prime}\right) & =p^{\ell} v_{\ell}\left(p, p^{\prime}\right)\left(p^{\prime}\right)^{\ell}
\end{aligned}
$$

and

$$
\tilde{G}_{\ell m, \ell^{\prime} m^{\prime}}\left(q^{\mathrm{on}}\right)=\frac{4 \pi}{L^{3}} \sum_{\vec{n}}\left(\frac{q}{q^{\mathrm{on}}}\right)^{\ell+\ell^{\prime}} Y_{\ell m}^{*}(\hat{q}) I(q) Y_{\ell^{\prime} m^{\prime}}(\hat{q}) .
$$

The factor $\left(q / q^{\text {on }}\right)^{\ell+\ell^{\prime}}$ can be replaced by 1 , when $\ell+\ell^{\prime}$ is even, and by $q / q^{\text {on }}$ otherwise [38], i.e.

$$
\begin{aligned}
\left(q / q^{\text {on }}\right)^{\ell+\ell^{\prime}} \rightarrow\left(q / q^{\text {on }}\right)^{k}, \\
k=0,1 \quad \text { for } \quad \ell+\ell^{\prime}=\text { even, odd } .
\end{aligned}
$$

Note that only even $\ell+\ell^{\prime}$ lead to non-zero contributions for $\vec{P}=\overrightarrow{0}$, as well as in case of equal-mass particle scattering.

Note also that if the factor $\left(q / q^{\text {on }}\right)$ is neglected one obtains expressions that are different from the original Lüscher approach [33, 34] not by exponentially suppressed terms, but terms suppressed as $1 / L^{4}$ [38]. In any case, we have checked numerically that effects for the results of this study, coming from the $\left(q / q^{\text {on }}\right)$ factor, are very small for the considered realistic box sizes.

An important remark is in order. At first glance, it seems that there is an ambiguity in the choice of $k$ in Eq. (28). Note, however, that this problem arose because one insisted on the on-shell prescription in the infinite volume limit. The above choice ensures that our finite-volume expressions are compatible with Lüscher's approach [33, 34] up to exponentially suppressed terms, and in the infinite-volume limit they are also compatible with the on-shell prescription. In particular, the quantity $\tilde{G}$ in the infinite-volume limit is replaced by

$$
\begin{gathered}
\tilde{G}_{\ell m, \ell^{\prime} m^{\prime}}\left(q^{\text {on }}\right) \underset{L \rightarrow \infty}{\longrightarrow} \delta_{\ell \ell^{\prime}} \delta_{m m^{\prime}} \int \frac{d^{3} \vec{q}}{(2 \pi)^{3}} I(q) \\
=\delta_{\ell \ell^{\prime}} \delta_{m m^{\prime}} G
\end{gathered}
$$

with $G$ from Eq. (4) (channel index omitted here). The Bethe-Salpeter equation in the infinite-volume limit takes the simple form given in Eq. (1), i.e.

$$
T_{\ell}\left(p, p^{\prime}\right)=V_{\ell}\left(p, p^{\prime}\right)+V_{\ell}\left(p, q^{\text {on }}\right) G\left(q^{\text {on }}\right) T_{\ell}\left(q^{\text {on }}, p^{\prime}\right)
$$

where the arguments are quoted explicitly. Finally, recall that the expressions in Eqs. (27) and (29) are defined with an implicit momentum cutoff at $q=q_{\max }$.

\section{Partial wave mixing with boost and multiple channels}

In Sec. IIC, we have considered the partial-wave expansion for a zero total momentum, $\vec{P}=\overrightarrow{0}$, of the two 
particles. Nothing changes conceptually if we consider moving frames instead, and the formalism of Sec. IA can be applied. In this case, the quantity $\tilde{G}$ is given by

$$
\begin{aligned}
\tilde{G}_{\ell m, \ell^{\prime \prime} m^{\prime \prime}} & =\frac{4 \pi}{L^{3}} \sum_{\vec{n}}^{\left|\vec{q}^{*}\right|<q_{\max }} \frac{E}{P_{0}}\left(\frac{q^{*}}{q^{\text {on } *}}\right)^{k} \\
& \times Y_{\ell m}^{*}\left(\hat{q}^{*}\right) Y_{\ell^{\prime \prime} m^{\prime \prime}}\left(\hat{q}^{*}\right) I\left(q^{*}\right) .
\end{aligned}
$$

Here, $\vec{q}^{*}$ is given by Eq. (13), $E \equiv \sqrt{s}$ is from Eq. (8), $k$ from Eq. (28), and $\tilde{G}_{\ell m, \ell^{\prime \prime} m^{\prime \prime}}$ is obviously the generalization of $\tilde{G}$ from Eq. (14) to higher partial waves. Note that, in case of particles with different masses, the states with even values of $\ell$ and odd values of $\ell^{\prime}$ can mix in a moving frame. In this case, as discussed above, we choose $k=1$.

In the large- $L$ limit, up to the terms exponentially suppressed in $L$, the quantity $\tilde{G}_{\ell m, \ell^{\prime \prime} m^{\prime \prime}}$ can be expressed in terms of Lüscher's zeta-functions. Namely,

$$
\tilde{G}_{\ell m, \ell^{\prime \prime} m^{\prime \prime}}-\delta_{\ell \ell^{\prime \prime}} \delta_{m m^{\prime \prime}} G=-\frac{q^{\mathrm{on} *}}{8 \pi \sqrt{s}} i^{\ell-\ell^{\prime \prime}} \mathcal{M}_{\ell m, \ell^{\prime \prime} m^{\prime \prime}}
$$

where $\mathcal{M}_{\ell m, \ell^{\prime \prime} m^{\prime \prime}}$ is given, e.g., by Eq. (39) of Ref. [73]. It is a linear combination of the Lüscher zeta-functions in the moving frame.

The discrete levels in a finite volume emerge at the energies where the determinant of the linear equation (25) vanishes,

$$
\operatorname{det}\left(\delta_{\ell \ell^{\prime}} \delta_{m m^{\prime}}-V_{\ell}\left(q^{\text {on }}, q^{\text {on }}\right) \tilde{G}_{\ell m, \ell^{\prime} m^{\prime}}\left(q^{\text {on }}\right)\right)=0 .
$$

Here, $\tilde{G}$ is a matrix with a row (column) index given by all combinations of $\ell, m\left(\ell^{\prime}, m^{\prime}\right)$.

Finally, all above formulae refer to the single-channel case. In case of multiple coupled channels, both the potential $V$ and the quantity $\tilde{G}$ should be considered as matrices in channel space. In that case, $V$, which is diagonal in the space of partial waves, obtains additional channel indices $V_{\ell} \rightarrow V_{\ell}^{(i j)}$. The quantity $\tilde{G}$, that has non-diagonal elements in the space of partial waves due to the mixing, becomes a diagonal matrix in channel space, $\tilde{G}_{\ell m, \ell^{\prime} m^{\prime}} \rightarrow \tilde{G}_{\ell m, \ell^{\prime} m^{\prime}}^{(i)}$, c.f. also Eq. (2).

In the following, along with UChPT, we shall use the Inverse Amplitude Method (IAM) in the version of Ref. 62] in the finite volume, as has been done already in Ref. [45] for the case $\vec{P}=0$. The IAM method exploits unitarity, which in one channel states that $\operatorname{Im} T^{-1}=p /(8 \pi E)$ (see Eq. (3)). Denoting by $V^{[2]}$ and $V^{[4]}$ the second- and fourth-order chiral potentials, and noting that the amplitude at lowest order, $V^{[2]} \equiv T^{[2]}$, has no imaginary part, a dispersion relation is made for the function $\left(V^{[2]}\right)^{2} / T$, where the imaginary part is thus known analytically. It leads to a simple relationship $T^{\mathrm{IAM}}=\left(T^{[2]}\right)^{2} /\left(T^{[2]}-T^{[4]}\right)$, where $T^{[2]}$, and $T^{[4]}$ are the amplitudes at lowest order and nextto-lowest order, respectively 68]. We can then recast these results in terms of Eq. (10), redefining a potential $V^{[4]}=T^{[4]}-V^{[2]} G V^{[2]}$. The equivalent potential to be used in Eq. (11) or (33) to obtain the results of the IAM amplitude is then given by

$$
V_{\ell}^{\mathrm{IAM}}=\left(1-V^{[4]}\left(V^{[2]}\right)^{-1}\right)^{-1} V^{[2]}
$$

Here, $V^{[2]} \equiv\left(V^{[2]}\right)^{(i j)}$ and $V^{[4]} \equiv\left(V^{[4]}\right)^{(i j)}$ are matrices in channel space. For $V^{[4]}$, only the polynomial terms are considered like in Ref. [62]. The method derived here could be also extended to the loop calculations of Refs. 69 72.

For illustration, an explicit example for the emerging structure is quoted. With $V_{\ell}^{(i j)} \equiv\left(V_{\ell}^{\text {IAM }}\right)^{(i j)}$, we consider the mixing of $S$ - and $P$-waves in the coupled channels $\pi K$ and $\eta K$. Then, $V$ and $\tilde{G}$ in Eq. (33) are given by

$$
\begin{aligned}
& V_{\ell}^{(i j)}= \\
& \left(\begin{array}{cccccccc}
V_{0}^{(11)} & V_{0}^{(12)} & 0 & 0 & 0 & 0 & 0 & 0 \\
V_{0}^{(21)} & V_{0}^{(22)} & 0 & 0 & 0 & 0 & 0 & 0 \\
0 & 0 & V_{1}^{(11)} & V_{1}^{(12)} & 0 & 0 & 0 & 0 \\
0 & 0 & V_{1}^{(21)} & V_{1}^{(22)} & 0 & 0 & 0 & 0 \\
0 & 0 & 0 & 0 & V_{1}^{(11)} & V_{1}^{(12)} & 0 & 0 \\
0 & 0 & 0 & 0 & V_{1}^{(21)} & V_{1}^{(22)} & 0 & 0 \\
0 & 0 & 0 & 0 & 0 & 0 & V_{1}^{(11)} & V_{1}^{(12)} \\
0 & 0 & 0 & 0 & 0 & 0 & V_{1}^{(21)} & V_{1}^{(22)}
\end{array}\right)
\end{aligned}
$$

and 


$$
\tilde{G}_{\ell m, \ell^{\prime} m^{\prime}}^{(i)}=\left(\begin{array}{cccccccc}
\tilde{G}_{00,00}^{(1)} & 0 & \tilde{G}_{00,1-1}^{(1)} & 0 & \tilde{G}_{00,10}^{(1)} & 0 & \tilde{G}_{00,11}^{(1)} & 0 \\
0 & \tilde{G}_{00,00}^{(2)} & 0 & \tilde{G}_{00,1-1}^{(2)} & 0 & \tilde{G}_{00,10}^{(2)} & 0 & \tilde{G}_{00,11}^{(2)} \\
\tilde{G}_{1-1,00}^{(1)} & 0 & \tilde{G}_{1-1,1-1}^{(1)} & 0 & \tilde{G}_{1-1,10}^{(1)} & 0 & \tilde{G}_{1-1,11}^{(1)} & 0 \\
0 & \tilde{G}_{1-1,00}^{(2)} & 0 & \tilde{G}_{1-1,1-1}^{(2)} & 0 & \tilde{G}_{1-1,10}^{(2)} & 0 & \tilde{G}_{1-1,11}^{(2)} \\
\tilde{G}_{10,00}^{(1)} & 0 & \tilde{G}_{10,1-1}^{(1)} & 0 & \tilde{G}_{10,10}^{(1)} & 0 & \tilde{G}_{10,11}^{(1)} & 0 \\
0 & \tilde{G}_{10,00}^{(2)} & 0 & \tilde{G}_{10,1-1}^{(2)} & 0 & \tilde{G}_{10,10}^{(2)} & 0 & \tilde{G}_{10,11}^{(2)} \\
\tilde{G}_{11,00}^{(1)} & 0 & \tilde{G}_{11,1-1}^{(1)} & 0 & \tilde{G}_{11,10}^{(1)} & 0 & \tilde{G}_{11,11}^{(1)} & 0 \\
0 & \tilde{G}_{11,00}^{(2)} & 0 & \tilde{G}_{11,1-1}^{(2)} & 0 & \tilde{G}_{11,10}^{(2)} & 0 & \tilde{G}_{11,11}^{(2)}
\end{array}\right)
$$

\section{E. Symmetries of Eq. (33)}

As mentioned above, rotational symmetry is broken down to the cubic group on a finite lattice, and a mixing of different partial waves occurs. In moving frames, the symmetry is further broken down to the different subgroups of the cubic group (different little groups). Using symmetry arguments, it is possible to carry out a partial diagonalization of Eq. (33), as well as to construct the operators that project out the spectra corresponding to the different irreducible representations of the little groups. A full-fledged analysis of the problem has been carried out recently 73]. For earlier work on the subject, see, e.g., Refs. 29, 34, 51, 56, 57, 74 and references therein. Below, we use the results of Ref. [73], in case of the meson-meson scattering in $S, P, D$ waves, in order to attribute the emerging structures to the different little groups.

$$
\text { 1. The case } \vec{P}=(2 \pi / L)(0,0,0)
$$

The partial waves $S, P, D$ do not mix - the pertinent part of the matrix $\tilde{G}$ is diagonal in $\ell, \ell^{\prime}$ and all entries with $m-m^{\prime} \neq 0 \bmod 4$ vanish. Non-vanishing elements are: $\tilde{G}_{00,00}, \tilde{G}_{11,11}=\tilde{G}_{10,10}=\tilde{G}_{1-1,1-1}, \tilde{G}_{21,21}=$ $\tilde{G}_{2-1,2-1}, \tilde{G}_{20,20}, \tilde{G}_{22,22}=\tilde{G}_{2-2,2-2}, \quad \tilde{G}_{22,2-2}=$ $\tilde{G}_{2-2,22}=\left(\tilde{G}_{20,20}-\tilde{G}_{21,21}\right) / 2$.

Below we list the irreducible representations for a given $\ell$ and the pertinent equations for the determination of the energy levels [34], obtained from Eq. (33):

$$
\begin{array}{lll}
\ell=0, & A_{1}^{+}: & 1-V_{0} \tilde{G}_{00,00}=0, \\
\ell=1, & T_{1}^{-}: & 1-V_{1} \tilde{G}_{10,10}=0, \\
\ell=2, & E^{+}: & 1-V_{2} \tilde{G}_{21,21}=0, \\
\ell=2, & T_{2}^{+}: & 1-V_{2} \tilde{G}_{20,20}=0 .
\end{array}
$$

Note that for more than one channel, $V_{\ell}$ and $\tilde{G}_{\ell m, \ell^{\prime} m^{\prime}}$ in these equations can be considered as matrices in channel space. See also Sec.IID The energy levels are then given by the zeros of the corresponding determinants. For the cases of boosts with $\vec{P} \neq 0$, one has to be slightly more careful as the order of the matrices matter, and the formulae of the following sections are derived for the onechannel case. An example of partial wave mixing with multiple channels is explicitly evaluated in Sec. IVA.

$$
\text { 2. The case } \vec{P}=(2 \pi / L)(0,0,1)
$$

In the unequal mass case, we restrict ourselves to $S, P$ waves only. This corresponds to the partial wave mixing studied for the $\kappa(800), K^{*}(892)$ system in Sec. III. The non-zero matrix elements of $\tilde{G}$ are: $\tilde{G}_{00,00}, \tilde{G}_{11,11}=$ $\tilde{G}_{1-1,1-1}, \tilde{G}_{10,10}, \tilde{G}_{00,10}=\tilde{G}_{10,00}$.

The irreducible representations and the equations for the determination of the energy levels are given by solving Eq. (33):

$$
\begin{array}{lll}
\ell=0,1, \quad A_{1}: \quad & \left(1-V_{0} \tilde{G}_{00,00}\right)\left(1-V_{1} \tilde{G}_{10,10}\right) \\
& -V_{0} V_{1} \tilde{G}_{00,10}^{2}=0, \\
\ell=1, \quad E: \quad 1-V_{1} \tilde{G}_{11,11}=0 .
\end{array}
$$

Note that here and in the following, the $\tilde{G}_{\ell m, \ell^{\prime} m^{\prime}}$ can be mapped to linear combinations of the Lüscher zetafunctions or the quantities $w_{\ell^{\prime \prime}} m^{\prime \prime}$ used, e.g., in Refs. 56, 57] (see Eq. (321)).

We shall need $D$-waves in the equal-mass case only. In particular, we will study the mixing of $D$-waves with $S$-waves for the $\sigma(600)$ isoscalar in Sec. IV] For equal masses, $\tilde{G}_{00,10}=0$ and the mixing between the $S$ - and $P$-waves vanishes. The mixing vanishes anyway for $\pi \pi$ as $L+I=$ even, and the $P$-wave has isospin 1 .

Additional non-zero matrix elements are: $\tilde{G}_{00,20}=$ $\tilde{G}_{20,00}, \tilde{G}_{20,20}, \tilde{G}_{22,22}=\tilde{G}_{2-2,2-2}, \tilde{G}_{21,21}=\tilde{G}_{2-1,2-1}$, $\tilde{G}_{22,2-2}=\tilde{G}_{2-2,22}$.

The irreducible representations and the equations for 
the determination of the energy levels are:

$$
\begin{array}{lll}
\ell=0,2, & A_{1}^{+}: \quad & \left(1-V_{0} \tilde{G}_{00,00}\right)\left(1-V_{2} \tilde{G}_{20,20}\right) \\
& & -V_{0} V_{2} \tilde{G}_{00,20}^{2}=0, \\
\ell=2, & E^{+}: & 1-V_{2} \tilde{G}_{21,21}=0, \\
\ell=2, & B_{1}^{+}: & 1-V_{2}\left(\tilde{G}_{22,22}+\tilde{G}_{22,2-2}\right)=0, \\
\ell=2, & B_{2}^{+}: & 1-V_{2}\left(\tilde{G}_{22,22}-\tilde{G}_{22,2-2}\right)=0 .
\end{array}
$$

\section{The case $\vec{P}=(2 \pi / L)(1,1,0)$}

For the unequal mass case, we again consider $S, P$ waves only. The non-zero matrix elements of $\tilde{G}$ are: $\tilde{G}_{00,00}, \tilde{G}_{00,1-1}, \tilde{G}_{00,11}=-i \tilde{G}_{00,1-1}, \tilde{G}_{1-1,00}=$ $i \tilde{G}_{00,1-1}, \quad \tilde{G}_{11,00}=-\tilde{G}_{00,1-1}, \quad \tilde{G}_{10,10}, \quad \tilde{G}_{11,11}=$ $\tilde{G}_{1-1,1-1}, \tilde{G}_{11,1-1}=-\tilde{G}_{1-1,11}$. The irreducible representations and the equations for the determination of the energy levels are:

$$
\begin{aligned}
& \ell=0,1, \quad A_{1}:\left(1-V_{0} \tilde{G}_{00,00}\right) \\
& \times\left(1-V_{1}\left(\tilde{G}_{1-1,1-1}-i \tilde{G}_{11,1-1}\right)\right) \\
&-V_{0} V_{1}\left(\tilde{G}_{00,11}-\tilde{G}_{00,1-1}\right)^{2}=0, \\
& \ell=1, \quad B_{1}: 1-V_{1} \tilde{G}_{10,10}=0, \\
& \ell=1, \quad B_{2}: 1-V_{1}\left(\tilde{G}_{1-1,1-1}+i \tilde{G}_{11,1-1}\right)=0 .
\end{aligned}
$$

The mixing of $S$ - and $D$-waves is again considered in the equal-mass case. The irreducible representations and the equations for the determination of the energy levels are:

$$
\begin{aligned}
& \ell=0,2, \quad A_{1}^{+}:\left(1-V_{0} \tilde{G}_{00,00}\right)\left[\left(1-V_{2} \tilde{G}_{20,20}\right)\right. \\
& \times\left(1-V_{2}\left(\tilde{G}_{2-2,2-2}-\tilde{G}_{2-2,22}\right)\right) \\
&\left.+2 V_{2}^{2} \tilde{G}_{2-2,20}^{2}\right] \\
&+2\left(1-V_{2} \tilde{G}_{20,20}\right) V_{0} V_{2} \tilde{G}_{00,2-2}^{2} \\
&-\tilde{G}_{00,20} V_{0} V_{2}\left[\tilde{G}_{00,20}\right. \\
& \times\left(1-V_{2} \tilde{G}_{2-2,2-2}+V_{2} \tilde{G}_{2-2,22}\right) \\
&\left.+4 V_{2} \tilde{G}_{00,2-2} \tilde{G}_{2-2,20}\right]=0, \\
& \quad A_{2}^{+}: 1-V_{2}\left(\tilde{G}_{2-1,2-1}-i \tilde{G}_{2-1,21}\right)=0, \\
& \ell=2, \quad B_{1}^{+}: 1-V_{2}\left(\tilde{G}_{2-1,2-1}+i \tilde{G}_{2-1,21}\right)=0, \\
& \ell=2, \quad B_{2}^{+}: 1-V_{2}\left(\tilde{G}_{2-2,2-2}+\tilde{G}_{2-2,22}\right)=0 . \\
& \ell=2, \quad
\end{aligned}
$$

$$
\text { 4. The case } \vec{P}=(2 \pi / L)(1,1,1)
$$

For the unequal mass case, we consider $S, P$ waves only. The non-zero matrix elements of $\tilde{G}$ are: $\tilde{G}_{00,00}$, $\tilde{G}_{00,1-1}=-\tilde{G}_{11,00}=2^{-1 / 2}(1-i) \tilde{G}_{00,10}, \tilde{G}_{1-1,00}=$ $-\tilde{G}_{00,11}=2^{-1 / 2}(1+i) \tilde{G}_{00,10} \cdot \tilde{G}_{00,10}=\tilde{G}_{10,00}$, $\tilde{G}_{1-1,1-1}=\tilde{G}_{10,10}=\tilde{G}_{11,11}, \tilde{G}_{11,1-1}=-\tilde{G}_{1-1,11}$, $\tilde{G}_{1-1,10}=-\tilde{G}_{10,11}=2^{-1 / 2}(1-i) \tilde{G}_{11,1-1}, \tilde{G}_{11,10}=$ $-\tilde{G}_{10,1-1}=2^{-1 / 2}(1+i) \tilde{G}_{11,1-1}$. The irreducible representations and the equations for the determination of the energy levels are:

$$
\begin{aligned}
& \ell=0,1, \quad A_{1}: \quad\left(1-V_{1}\left(\tilde{G}_{1-1,1-1}+2 i \tilde{G}_{1-1,11}\right)\right) \\
& \times\left(1-V_{0} \tilde{G}_{00,00}\right)-3 V_{0} V_{1} \tilde{G}_{00,10}^{2}=0, \\
& \ell=1, \quad E: \quad 1-V_{1}\left(\tilde{G}_{1-1,1-1}-i \tilde{G}_{1-1,11}\right)=0 .
\end{aligned}
$$

The mixing of $S$ - and $D$-waves is considered in the equal-mass case. The irreducible representations and the equations for the determination of the energy levels are:

$$
\begin{aligned}
\ell=0,2, \quad A_{1}^{+}: \quad & \frac{V_{0} V_{2}}{10}\left(4 \sqrt{3} \tilde{G}_{2-2,20}+3 \sqrt{2} \tilde{G}_{2-1,21}\right)^{2} \\
& +\left(1-V_{2}\left(\tilde{G}_{2-1,2-1}+2 i \tilde{G}_{2-1,21}\right)\right) \\
& \times\left(1-V_{0} \tilde{G}_{00,00}\right)=0 \\
\ell=2, \quad E^{+}: \quad & \left(1-V_{2} \tilde{G}_{20,20}\right) \\
& \left(1-V_{2}\left(\tilde{G}_{2-1,2-1}-i \tilde{G}_{2-1,21}\right)\right) \\
& -6 V_{2}^{2} \tilde{G}_{2-1,20} \tilde{G}_{20,2-1}=0 .
\end{aligned}
$$

As mentioned before, our results completely agree with those of Ref. 73] in all partial waves. Further, note that in Ref. [57], the equations are derived for the cases $\vec{P}=(2 \pi / L)(0,0,1)$ and $\vec{P}=(2 \pi / L)(1,1,0)$, only $S$ and $P$ waves retained. We have checked that our results exactly agree with the results of Ref. [57] for these cases (the representations $B_{1}, B_{2}$ for $\vec{P}=(2 \pi / L)(1,1,0)$ are denoted by $B_{3}, B_{2}$ in Ref. [57]). In Ref. [56], results for the boosts $P=(2 \pi / L)(0,0,1)$ and $\vec{P}=(2 \pi / L)(1,1,0)$ have been obtained, and in Ref. [58] results equivalent to the present ones for the $S D$-wave case were determined.

\section{F. Calculation of the energy levels}

In practical calculations, we found it convenient to consider Eq. (33) in a rotated frame. We namely rotate the $\hat{z}$ direction of the coordinate system, in which $(\theta, \phi)$ of $\vec{q}^{*}$ are measured, into the direction of the boost vector $\vec{P}$. To rotate $\hat{z}$ actively into the $\vec{P}$ direction - the latter given by $\left(\theta_{P}, \phi_{P}\right)$ - one has different choices; we choose here first a rotation around the $\hat{x}$-axis by $-\theta_{P}$, so that the new $\hat{z}^{\prime}$ vector is in the $y z$ plane, and then a rotation around the original $\hat{z}$-axis by $\phi_{P}-\pi / 2$ so that $\hat{z}^{\prime \prime}|| \vec{P}$. 
The coordinates $\vec{q}^{* \prime \prime}$ of a vector $\vec{q}^{*}$, in the rotated coordinate system with $z$-axis $\hat{z}^{\prime \prime}$, are then given by the inverse of these (non-commuting) rotations. With standard rotation matrices $R_{i}, i=x, y, z$, the new Cartesian coordinates read

$$
\begin{aligned}
& \vec{q}^{* \prime \prime}=R \vec{q}^{*}=\left(R_{z}\left(\phi_{P}-\frac{\pi}{2}\right) R_{x}\left(-\theta_{P}\right)\right)^{-1} \vec{q}^{*}, \\
& R=\left(\begin{array}{ccc}
\sin \phi_{P} & -\cos \phi_{P} & 0 \\
\cos \theta_{P} \cos \phi_{P} & \cos \theta_{P} \sin \phi_{P} & -\sin \theta_{P} \\
\sin \theta_{P} \cos \phi_{P} & \sin \theta_{P} \sin \phi_{P} & \cos \theta_{P}
\end{array}\right) \text {. }
\end{aligned}
$$

where

$$
\begin{array}{lll}
\theta_{P}=0, & \phi_{P}=0 & \text { for } P=(2 \pi / L)(0,0,1), \\
\theta_{P}=\pi / 2, & \phi_{P}=\pi / 4 & \text { for } P=(2 \pi / L)(1,1,0), \\
\theta_{P}=\arctan \sqrt{2}, & \phi_{P}=\pi / 4 & \text { for } P=(2 \pi / L)(1,1,1) .
\end{array}
$$

We would like to stress that all calculations in this paper have been carried out in the rotated frame. The formulae listed in the previous section merely serve to demonstrate the equivalence of the present approach to that of Ref. 73.

From now on we will only consider the $\tilde{G}$ functions in the rotated coordinate system (compare to Eq. (31)),

$$
\begin{aligned}
& \tilde{G}_{\ell m, \ell^{\prime} m^{\prime}}^{R}=\frac{4 \pi}{L^{3}} \sum_{\vec{n}}^{\left|\vec{q}^{*}\right|<q_{\max }} \frac{E}{P_{0}}\left(\frac{q^{*}}{q^{\text {on } *}}\right)^{k} \\
& \times Y_{\ell m}^{*}\left(\hat{q}^{* \prime \prime}\left(\hat{q}^{*}\right)\right) Y_{\ell^{\prime} m^{\prime}}\left(\hat{q}^{* \prime \prime}\left(\hat{q}^{*}\right)\right) I\left(q^{*}\right) .
\end{aligned}
$$

In particular, with the rotation of the coordinate system the zeros of the determinant, i.e. the levels, remain unchanged and the matrix of Eq. (33) becomes blockdiagonal.

For the boosts $\vec{P}=(2 \pi / L)(0,0,0),(2 \pi / L)(0,0,1)$, $(2 \pi / L)(0,1,0), \quad(2 \pi / L)(1,0,0), \quad(2 \pi / L)(0,0,2), \quad \cdots$, Eqs. (37), (38), (39) remain of course valid with $G^{R}$ from Eq. (46), because for these boosts $\tilde{G}_{\ell m, \ell^{\prime} m^{\prime}}^{R}=\tilde{G}_{\ell m, \ell^{\prime} m^{\prime}}$. For the boost $\vec{P}=(2 \pi / L)(1,1,0)$, we obtain:

$$
\begin{array}{lll}
\ell=0,1, & A_{1}: & \left(1-V_{0} \tilde{G}_{00,00}^{R}\right)\left(1-V_{1} \tilde{G}_{10,10}^{R}\right) \\
& & -V_{0} V_{1}\left(\tilde{G}_{00,10}^{R}\right)^{2}=0, \\
\ell=1, & B_{1}: & 1-V_{1}\left(\tilde{G}_{11,11}^{R}+\tilde{G}_{11,1-1}^{R}\right)=0, \\
\ell=1, & B_{2}: & 1-V_{1}\left(\tilde{G}_{11,11}^{R}-\tilde{G}_{11,1-1}^{R}\right)=0, \\
\ell=2, & A_{2}^{+}: & 1-V_{2}\left(\tilde{G}_{22,2-2}^{R}-\tilde{G}_{22,22}^{R}\right)=0, \\
\ell=2, & B_{1}^{+}: \quad 1-V_{2}\left(\tilde{G}_{21,21}^{R}+\tilde{G}_{21,2-1}^{R}\right)=0, \\
\ell=2, & B_{2}^{+}: \quad 1-V_{2}\left(\tilde{G}_{21,21}^{R}-\tilde{G}_{21,2-1}^{R}\right)=0, \\
\ell=0,2, & A_{1}^{+}: \quad 1-V_{0} \tilde{G}_{00,00}^{R}-a V_{2}+b V_{2}^{2}=0
\end{array}
$$

where

$$
\begin{aligned}
a= & \tilde{G}_{2-2,2-2}^{R}+\tilde{G}_{2-2,22}^{R}+\tilde{G}_{20,20}^{R} \\
+ & V_{0}\left[2\left(\tilde{G}_{00,2-2}^{R}\right)^{2}+\left(\tilde{G}_{00,20}^{R}\right)^{2}\right. \\
& \left.-\tilde{G}_{00,00}^{R}\left(\tilde{G}_{2-2,2-2}^{R}+\tilde{G}_{2-2,22}^{R}+\tilde{G}_{20,20}^{R}\right)\right] \\
b= & 2\left(\tilde{G}_{2-2,20}^{R}\right)^{2}\left(V_{0} \tilde{G}_{00,00}^{R}-1\right) \\
- & 4 V_{0} \tilde{G}_{00,2-2}^{R} \tilde{G}_{00,20}^{R} \tilde{G}_{2-2,20}^{R} \\
+ & \left(\tilde{G}_{2-2,2-2}^{R}+\tilde{G}_{2-2,22}^{R}\right) \tilde{G}_{20,20}^{R} \\
+ & V_{0}\left[2 \tilde{G}_{20,20}^{R}\left(\tilde{G}_{00,2-2}^{R}\right)^{2}\right. \\
& \left.+\left(\tilde{G}_{2-2,2-2}^{R}+\tilde{G}_{2-2,22}^{R}\right)\left(\left(\tilde{G}_{00,20}^{R}\right)^{2}-\tilde{G}_{00,00}^{R} \tilde{G}_{20,20}^{R}\right)\right] .
\end{aligned}
$$

For the boost $\vec{P}=(2 \pi / L)(1,1,1)$, we obtain:

$$
\begin{array}{lrl}
\ell=0,1, \quad A_{1}: \quad\left(1-V_{0} \tilde{G}_{00,00}^{R}\right)\left(1-V_{1} \tilde{G}_{10,10}^{R}\right) \\
& -V_{0} V_{1}\left(\tilde{G}_{00,10}^{R}\right)^{2}=0, \\
\ell=1, \quad E: \quad 1-V_{1} \tilde{G}_{11,11}^{R}=0, \\
\ell=0,2, \quad A_{1}^{+}: \quad\left(1-V_{0} \tilde{G}_{00,00}^{R}\right)\left(1-V_{2} \tilde{G}_{20,20}^{R}\right) \\
& -V_{0} V_{2}\left(\tilde{G}_{00,20}^{R}\right)^{2}=0, \\
\ell=2, \quad E^{+}: \quad\left(1-V_{2} \tilde{G}_{22,22}^{R}\right)\left(1-V_{2} \tilde{G}_{21,21}^{R}\right) \\
\quad & -V_{2}^{2} \tilde{G}_{22,2-1}^{R} \tilde{G}_{2-1,22}^{R}=0 .
\end{array}
$$

Finally, we denote that the structures of Eq. (36) are the same with rotated $\tilde{G}^{R}$, except that the rotation renders many $\tilde{G}^{R}$ equal to zero, so that the block-diagonal structure becomes immediately visible.

\section{G. Equations for the determination of the scattering phase shifts}

One of the main tasks of this study is the reconstruction of the $S$-wave phase shift from lattice data taken in moving frames. We will assume that suitable interpolating operators are used in lattice simulations that allow to associate the levels obtained to any of the symmetry groups discussed. A step forward in this direction has been given, e.g., in Refs. [58, 73] by constructing a basis of interpolating operators transforming irreducibly under the reduced symmetry of the moving particle in a cubic box. Similarly, a complete list of two-particle interpolators is quoted in Ref. [57] for meson momenta $p_{i}$ where $p_{1}, p_{2} \leq 2 \pi \sqrt{3} / L$.

The structure of the equations for the level determination, denoted in the previous section, lends itself to 
a suitable strategy: the $P$ - or $D$-waves can be reconstructed separately and serve as input to disentangle the $S$-wave from the representations $A_{1}$ and $A_{1}^{+}$in which $P$ or $D$-waves appear mixed with the $S$-wave, respectively. For the one-channel case, a corresponding set of equations is formulated in the following.

Note that to disentangle partial waves one needs in principle eigenvalues from different irreducible representations at exactly the same energy, which will unlikely be the case in an actual lattice calculation. This requires to make assumptions on the continuity of the amplitude so that one can interpolate to different energies. Strategies of how to do this in practice are discussed in Sec. IV B 1.

The key point for phase extraction is that, although Eqs. (38, 39, 47) and (49) are formulated in terms of the divergent, and thus cut-off dependent $\tilde{G}$, the extraction and disentanglement of partial waves can be formulated entirely in terms of

$$
\begin{aligned}
\hat{G}_{\ell m, \ell^{\prime} m^{\prime}} & =\tilde{G}_{\ell m, \ell^{\prime} m^{\prime}}^{R}-\delta_{\ell \ell^{\prime}} \delta_{m m^{\prime}}\left(G+\frac{i p}{8 \pi E}\right) \\
& =\tilde{G}_{\ell m, \ell^{\prime} m^{\prime}}^{R}-\delta_{\ell \ell^{\prime}} \delta_{m m^{\prime}} \operatorname{Re} G
\end{aligned}
$$

with $\tilde{G}^{R}$ from Eq. (46) and $G$ from Eq. (41) (the last line of Eq. (50) is valid above threshold). For the quantity $\hat{G}$ the dependence on the cut-off cancels because it depends only on the difference $\tilde{G}-G$; the quantity $\tilde{G}$ for the case $\delta_{\ell \ell^{\prime}} \delta_{m m^{\prime}}=0$ is convergent anyway: for $\vec{q}^{*}$ much larger than the considered typical momenta, the sum (c.f. Eq. (46)) can be approximated by the integral and then Eq. (19) renders the high-momenta contributions to zero.

Below we demonstrate in one example, how to disentangle $S$-waves from the $P$-waves. We consider the irreducible representation $A_{1}$. Eqs. (38), (47) and (49) all have the same form in this representation

$$
\left(1-V_{0} \tilde{G}_{00,00}^{R}\right)\left(1-V_{1} \tilde{G}_{10,10}^{R}\right)-V_{0} V_{1}\left(\tilde{G}_{00,10}^{R}\right)^{2}=0 .
$$

This equation determines the $A_{1}$ levels for the boosts $\vec{P} \sim(0,0,1),(1,1,0),(1,1,1)$. We further introduce

$$
\begin{aligned}
T_{i} & =\frac{V_{i}}{1-V_{i} G}=\frac{-8 \pi E}{p \cot \delta_{i}(p)-i p}, \\
K_{i} & =\frac{V_{i}}{1-V_{i} \operatorname{Re} G}=\frac{-8 \pi E}{p \cot \delta_{i}(p)}, \quad i=0,1 .
\end{aligned}
$$

With these definitions, Eq. (51) is rewritten as

$$
\left(1-K_{0} \hat{G}_{00,00}\right)\left(1-K_{1} \hat{G}_{10,10}\right)-K_{0} K_{1}\left(\hat{G}_{00,10}\right)^{2}=0 .
$$

Solving this equation with respect to the $S$-wave $\delta_{0} \equiv \delta_{S}$ as a function of the $P$-wave $\delta_{1} \equiv \delta_{P}$, we finally obtain

$$
p \cot \delta_{0}=-8 \pi E \hat{G}_{00,00}+\frac{(8 \pi E)^{2} \hat{G}_{00,10}^{2}}{p \cot \delta_{1}+8 \pi E \hat{G}_{10,10}},
$$

which can also be obtained from Eq. (51) upon substitution of $\tilde{G}^{R}$ by $\hat{G}$ where $\hat{G}$ is defined in Eq. (50). Note that, although in the derivation of Eq. (54) we have used the quantities $\tilde{G}$ and $G$, the final result depends only on the cut-off independent quantity $\hat{G}$, as has to be.

If the $P$-wave phase shift vanishes, Eq. (54) simplifies to

$$
p \cot \delta_{0}=-8 \pi E \hat{G}_{00,00},
$$

i.e. the standard Lüscher formula for pure $S$-wave, in the formulation of Ref. [32].

Eq. (54) provides - in the one-channel case - the possibility to fully correct for partial wave mixing at the energy $E$ of the measured level, but it requires the knowledge of the $P$-wave phase shift $\delta_{1}$ at that energy. As discussed above, this knowledge may come from a separate analysis of levels with pure $P$-wave content, i.e. from other representations than $A_{1}$, c.f. Eqs. (38), (47) and (49). The following cases allow for this extraction of the $P$-wave: for $\vec{P}=(2 \pi / L)(0,0,0)$ : representation $T_{1}^{-}$, c.f. Eq. (37); for $\vec{P}=(2 \pi / L)(0,0,1)$ : representation $E$, c.f. Eqs. (38); for $\vec{P}=(2 \pi / L)(1,1,0)$ : representations $B_{1}$ and $B_{2}$, c.f. Eq. (47); for $\vec{P}=(2 \pi / L)(1,1,1)$ : representation $E$, c.f. Eq. (49).

Finally, we consider the mixing of $S$ - and $D$-waves in case of equal-mass particles. Extraction of the $D$-waves alone proceeds analogously to the extraction of the $P$ waves in all cases except $\vec{P}=(2 \pi / L)(1,1,1)$. In this case, there are two independent solutions

$$
\begin{aligned}
& p \cot \delta_{2}=-4 \pi E\left[\hat{G}_{22,22}+\hat{G}_{21,21}\right. \\
& \quad \pm \sqrt{\left.4 \hat{G}_{22,2-1} \hat{G}_{2-1,22}+\left(\hat{G}_{22,22}-\hat{G}_{21,21}\right)^{2}\right]} .
\end{aligned}
$$

This is related to the fact that, in this case, there are two different representations $E^{+}$for $\ell=2$.

Finally, the mixing of the $S$ - and $D$-waves can be treated analogously to the mixing of the $S$ - and $P$-waves, since the equations are always linear in $\cot \delta_{0}$. In the case $\vec{P}=(2 \pi / L)(1,1,0)$ the final equations are a bit cumbersome and contain terms quadratic in $\cot \delta_{2}$ (not explicitly quoted here).

For the two-channel case, the structure of the determinant (33) is more complicated, because the $V$ and $\tilde{G}$ are matrices in channel space as discussed in Sec. IID There is not much point to formulate analytic formulae as in this section, because for a disentanglement of the $S$-wave in a channel, one needs to know not only the $P$ or $D$-wave phase shift, but also inelasticities and phase shifts in the other channel. For $S$-wave and $\vec{P}=\overrightarrow{0}$, the reconstruction of the amplitude in the two-channel case has been discussed and solved in Ref. [32]. In this study, we will construct synthetic data using the full two-channel case and reconstruct the one-channel phase shifts using the framework developed in this section. Below the inelastic threshold, this provides a very good approximation whose validity will be discussed. 


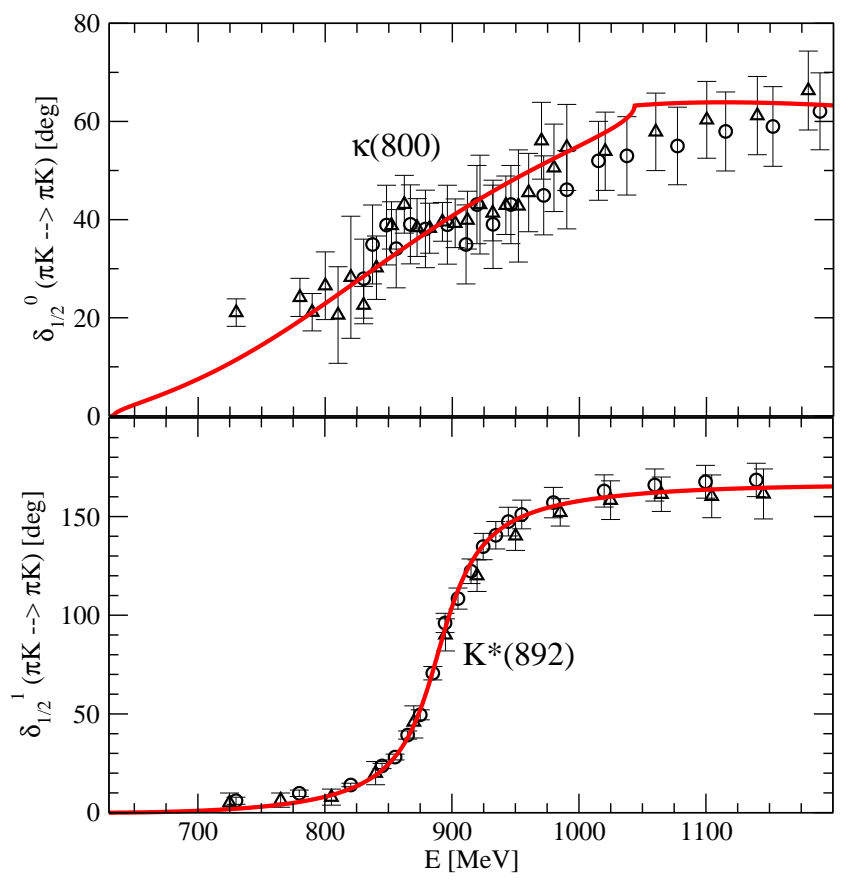

FIG. 1: Solution for the isospin $I=1 / 2$, strangeness $S=-1$ meson-meson interaction (solid lines) of Ref. [45]. Partial wave data for $\delta_{1 / 2}^{0}$ : circles [75], triangles up: average as defined in ref. [76]. For $\delta_{1 / 2}^{1}$ : triangles up [77], circles [78]

\section{THE $\kappa(800) / \mathrm{K}^{*}(892)$ SYSTEM}

\section{A. Level spectrum}

To study the mixing of partial waves and apply the framework developed in previous sections, the case of coupled-channel $\pi K, \eta K$ scattering in a moving frame is considered. In isospin $I=1 / 2$, strangeness $S=-1$, we take account of the $S$-wave with the $\kappa(800)$ resonance, the $P$-wave with the $K^{*}(892)$, and neglect all higher partial waves. $S$-wave $\pi K$ scattering and the $\kappa$ resonance have been addressed in recent lattice calculations, e.g. in Refs. 26, 27.

For the hadronic amplitude in the infinite volume, we use the solution obtained in Ref. [45] by fitting the low energy constants $L_{1}$ to $L_{8}$ to strangeness $S=0,-1$ partial wave data in $S$ - and $P$-waves. That solution is obtained using the inverse amplitude method of Ref. [62]. In Eq. (34) the connection to the present framework is quoted. The phase shifts are shown in Fig. 1] For the $S$-wave we observe a pronounced effect due to the $\eta K$ threshold which shows that this channel can have noticeable influence. For the $P$-wave, the $\eta K$ channel plays almost no role.

Using the equations of Sec. IE trum for the different boosts can be predicted as a function of the box length $L$. For $\vec{P}=\overrightarrow{0}$, the result is shown in Fig. 2 Here, $S$ - and $P$-waves do not mix. We ob-

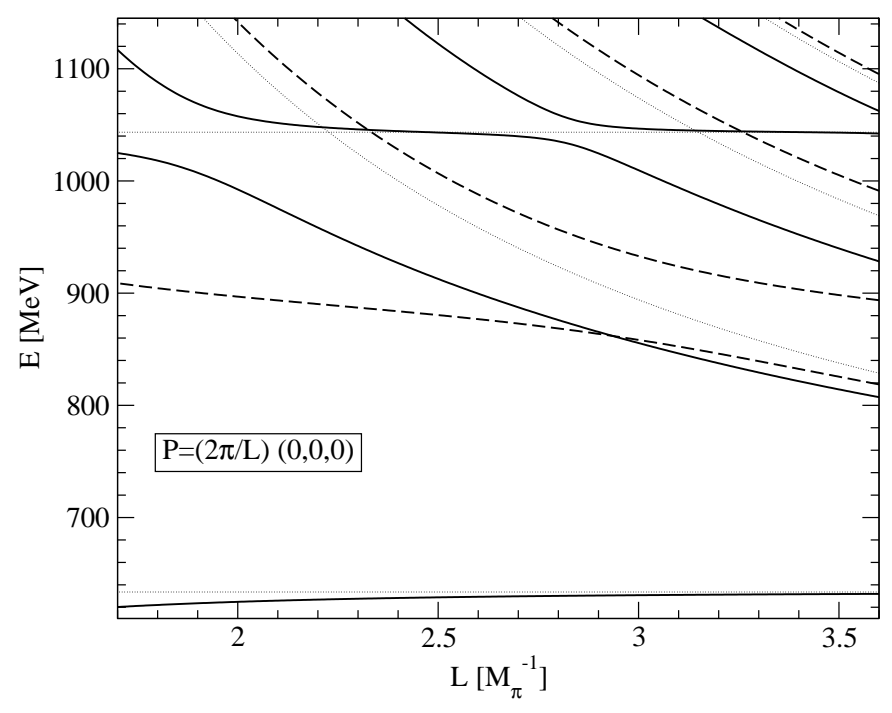

FIG. 2: Spectrum of the $S$ - and $P$-wave system in coupledchannel scattering $(\pi K, \eta K)$ for $\vec{P}=(0,0,0)$. Solid (dashed) lines: $S$-wave $(P$-wave). The fine dotted lines show the noninteracting levels.

serve an $S$-wave level close to the $\pi K$ threshold as well as avoided level crossing at the $\eta K$ threshold for the $S$ wave. These features have been discussed extensively in Refs. [32, 45]. In particular, the avoided level crossing is a signal of the $\eta K$ threshold (c.f. fine dashed horizontal line) and not of the $\kappa(800)$.

As we choose a finite boost $\vec{P} \neq \overrightarrow{0}$, the level spectrum becomes more complex. For $P=(2 \pi / L)(0,0,1)$, $P=(2 \pi / L)(1,1,0)$, and $P=(2 \pi / L)(1,1,1)$ the resulting levels as a function of $L$ are shown in Figs. 3, 4, and 5. respectively.

As the boost increases, the (non-interacting) levels are in general moved upwards in energy. Close to the lowest boosted non-interacting level, i.e. the boosted $\pi K$ threshold, one always finds a level in which $S$ - and $P$ waves are mixed (solid lines, representation $\left.A_{1}\right)^{1}$. At higher energies, apart from levels of this kind, also levels from other representations start to appear containing only $P$-wave without $S$-wave admixture and allowing for a separate analysis of the $P$-wave.

\footnotetext{
1 The levels of the representation $A_{1}$ are determined from Eq. (51). The first term of Eq. (51) suggests the occurrence of two levels that undergo a mixing through the second term $\sim V_{0} V_{1}$. However, as e.g. Fig. 3 shows, there is only one level close to the first non-interacting energy, and only one close to the second noninteracting energy. Indeed, it can be shown that this must be the case, by using the known angular structure of the $\hat{G}_{\ell m, \ell^{\prime} m^{\prime}}$.
} 


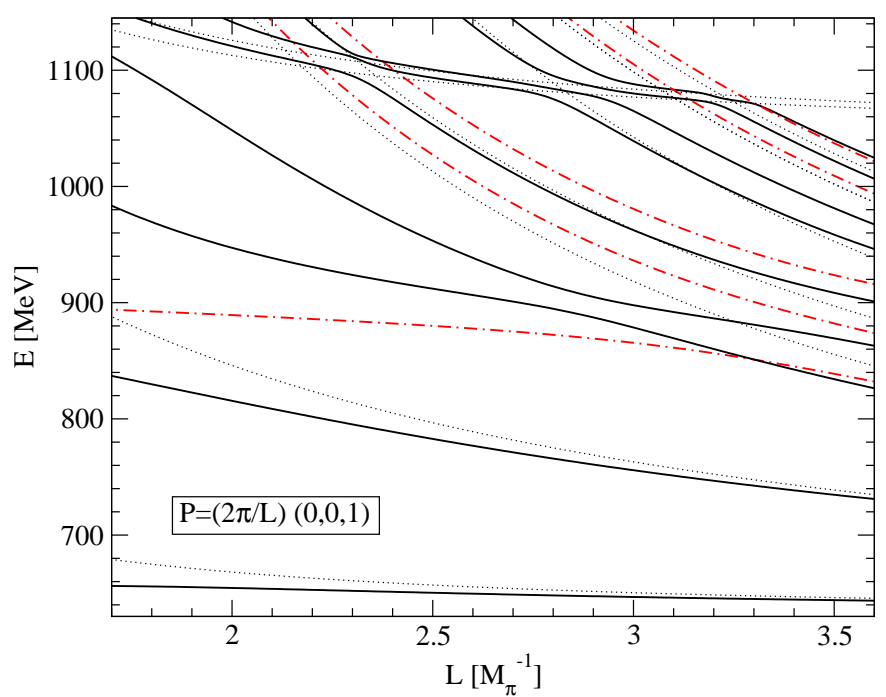

FIG. 3: Spectrum of the $S$ - and $P$-wave system in coupledchannel scattering $(\pi K, \eta K)$ for $\vec{P}=(2 \pi / L)(0,0,1)$. Solid lines: levels with $S P$-wave mixing from the irreducible representation $A_{1}$. Dash-dotted (red) lines: levels from only $P$ wave, irreducible representation $E$. The fine dotted lines show the boosted non-interacting levels.

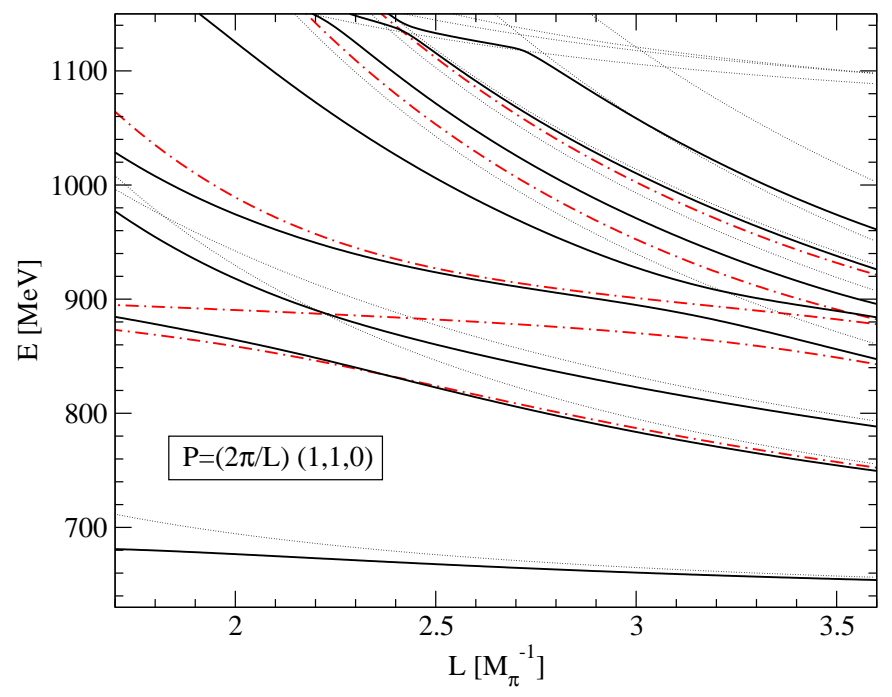

FIG. 4: Spectrum of the $S$ - and $P$-wave system in coupledchannel scattering $(\pi K, \eta K)$ for $\vec{P}=(2 \pi / L)(1,1,0)$. Solid lines: levels with $S P$-wave mixing from the irreducible representation $A_{1}$. Dash-dotted (red) lines: levels from only $P$ wave, irreducible representations $B_{1}$ and $B_{2}$. The fine dotted lines show the boosted non-interacting levels.

\section{B. Leading behavior of the level shifts}

The levels from the $A_{1}$ representation get shifted once the mixing is taken into account. To understand this effect, we consider mixing in the limit of small phase shifts. In the absence of a $P$-wave $\left(V_{1}=0\right)$, Eq. (51)

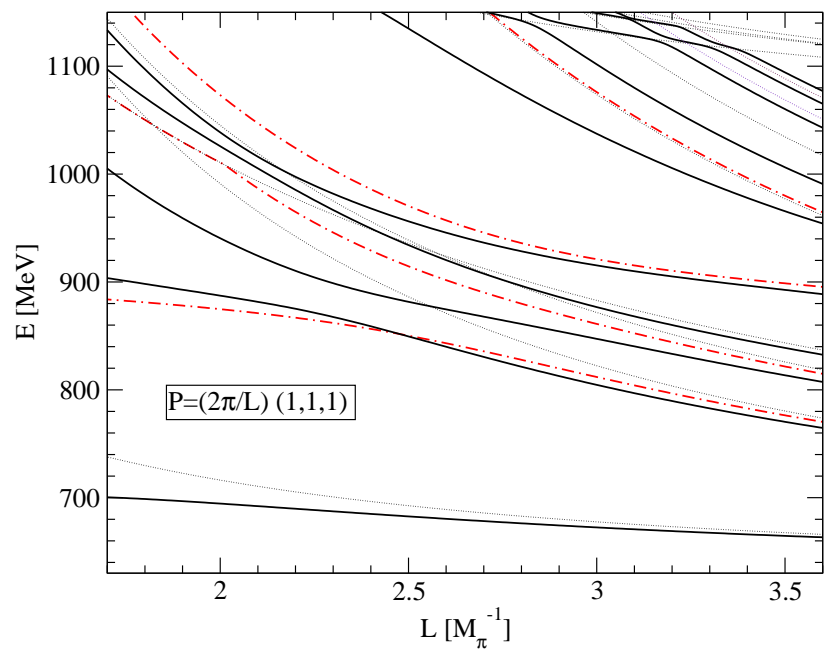

FIG. 5: Spectrum of the $S$ - and $P$-wave system in coupledchannel scattering $(\pi K, \eta K)$ for $\vec{P}=(2 \pi / L)(1,1,1)$. Solid lines: levels with $S P$-wave mixing from the irreducible representation $A_{1}$. Dash-dotted (red) lines: levels from only $P$ wave, irreducible representation $E$. The fine dotted lines show the boosted non-interacting levels.

reduces to

$$
1-V_{S} \tilde{G}_{00,00}^{R}\left(E=E_{S}\right)=0
$$

which determines the position $E_{S}$. The level shift is

$$
\Delta E=E_{S P}-E_{S}
$$

with $E_{S P}$ the position of the level with mixing, given by the solution of Eq. (51). The shift can be approximately calculated by Taylor expanding Eq. (51) around $E=E_{S}$. It is straightforward to show that then approximately

$$
\Delta E \simeq \delta_{P} \frac{8 \pi E_{S}}{p} \frac{\left(\hat{G}_{00,10}^{R}\right)^{2}}{\partial \hat{G}_{00,00}^{R} / \partial E}
$$

where $\delta_{P}$ is the $P$-wave phase shift, $p$ the threemomentum of the $\pi$ and $K$ in the two-particle rest frame, and $\hat{G}$ defined in Eq. (50). The actual shift $\Delta E$ is shown with the solid lines in Fig. 6, the result from Eq. (59) is indicated with the dashed lines. Indeed, for the lowest level where $\delta_{1}$ is small Eq. (59) provides a good approximation.

To understand the structure of the shift, we can expand $\hat{G}$ around the pole at $E=E_{0}$ where $E_{0}$ is the boosted non-interacting energy,

$$
\begin{aligned}
\hat{G}_{\ell m, \ell^{\prime} m^{\prime}}^{R} & =\frac{1}{L^{3}} \sum_{\vec{n}} h_{\ell m, \ell^{\prime} m^{\prime}}^{(\vec{n})} I\left(q^{*}\right) \\
& =\frac{a_{-1} h_{\ell m, \ell^{\prime} m^{\prime}}^{(\vec{n}=\overrightarrow{0}}}{E-E_{0}}+R_{\ell m, \ell^{\prime} m^{\prime}}
\end{aligned}
$$




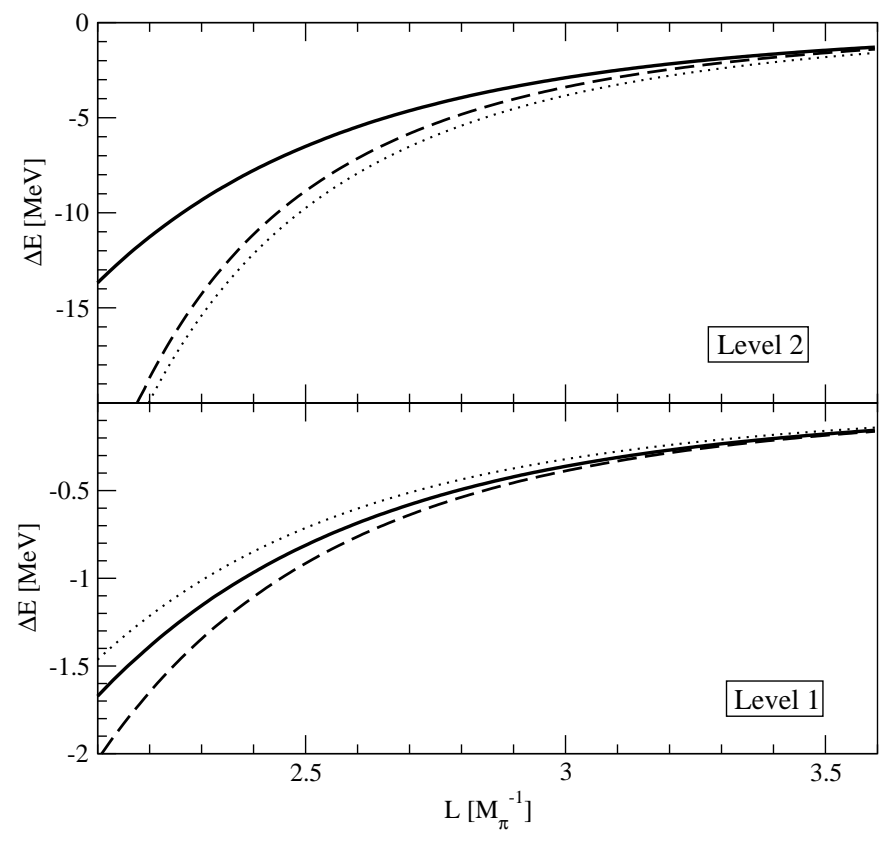

FIG. 6: Level shift through partial wave mixing for $\vec{P}=$ $(2 \pi / L)(0,0,1)$. Below: for the lowest level from Fig. 3 Above: for the following level. The solid lines show the actual level shift, the dashed lines the result of Eq. (59) and the dotted lines the leading behavior from Eq. (62).

where $h_{\ell m, \ell^{\prime} m^{\prime}}^{(\vec{n})}$ contains the angular structure, $a_{-1} h_{\ell m, \ell^{\prime} m^{\prime}}^{(\vec{n}=\overrightarrow{0}}$ is the residue, and $R_{\ell m, \ell^{\prime} m^{\prime}}$ is regular in the vicinity of $E_{0}$. The residue is readily evaluated,

$$
a_{-1}=\frac{1}{L^{3}} \frac{1}{2 \omega_{1} \omega_{2}} \frac{\omega_{1}+\omega_{2}}{E+\omega_{1}+\omega_{2}}
$$

where the energies $\omega_{i}$ are evaluated with the boosted vectors $\vec{q}^{*} \equiv \vec{q}^{*}\left(\vec{q}=\frac{2 \pi}{L}(0,0,0)\right)$ for the lowest level and $\vec{q}^{*}\left(\vec{q}=\frac{2 \pi}{L}(0,0,1)\right)$ for the following one. Using $\omega_{1}+\omega_{2} \simeq E$ and substituting Eq. (60) in Eq. (59) we obtain

$$
\Delta E \simeq-\frac{6 \pi E_{S} \delta_{P}}{L^{3} p \omega_{1} \omega_{2}}
$$

for the level shift. The result of this approximation is shown with the dotted lines in Fig. 6. This equation shows that the shift is to leading order in $E$ proportional to $\delta_{P}$ and to $1 / L^{-3}$. This $L^{-3}$ behavior is similar to the one of the scattering length [33, 45]. While Eq. (62) is useful to understand the qualitative behavior of the level shift, its quantitative use is limited.

\section{Disentangling the $\kappa(800) / K^{*}(892)$ system}

The $S$-wave can be extracted from lattice data of the representations $A_{1}^{+}$(boost $\vec{P}=\overrightarrow{0}$ ) and $A_{1}$ (boosts $\vec{P}=(2 \pi / L)(0,0,1), \quad(2 \pi / L)(0,0,1), \quad(2 \pi / L)(1,1,0)$,
$(2 \pi / L)(1,1,1))$. While for $\vec{P}=\overrightarrow{0}$ the $S$-wave does not mix with the $P$-wave, for the higher boosts mixing occurs in the $A_{1}$ representation, c.f. Eqs. (37, 38, 47, 49). We have derived Eq. (54) that allows to disentangle the $S$-wave for the one-channel problem provided that the $P$-wave is known from an analysis of other levels, as discussed in Sec. II G.

In this section, we apply Eq. (54) to the levels corresponding to $A_{1}$ shown in Figs. 3 to 5 . It should be stressed that those levels have been obtained with the full two-channel formalism as described in Sec. IID based on the global fit of low energy constants to $S$ - and $P$-wave partial wave data as described in Sec. II A. In contrast, we will extract the phase shift from these levels using the one-channel equation (54). Below the inelastic threshold, given by the $\eta K$ channel, this is expected to be a good approximation. Indeed, in Refs. [31, 32], the socalled pseudo-phase, i.e. the phase extracted with a onechannel formalism from a two-channel problem, provides an excellent approximation to the actual phase up to energies close below the inelastic threshold.

In Fig. 7. the actual $\pi K S$-wave phase shift, from the full coupled-channel system, is shown with the solid (orange) lines, identical to the corresponding curve in Fig. 1. Consider the first two $A_{1}$ levels, tied to the first three boosts [solid (black) lines in Figs. 3] 4, and 5]. In those levels, the $S$-wave mixes with the $P$-wave, and Eq. (54) provides the possibility to disentangle the $S$-wave by using the known $P$-wave shown in Fig. 1. The result is shown with the long-dashed lines in Fig. 17. In the figure, we also indicate to which box size $L$ the extracted phase shift corresponds (c.f. again Figs. 3, 4, and 5 to see the connection of energies and box size, given by the levels). As a test of the formalism, we have shown that once levels are generated from the hadronic model in a reduction to one channel, Eq. (54) ensures the exact reconstruction of the phase, as must be.

In general, the agreement with the original phase shift is excellent. Only at higher energies there are small deviations, coming from the more and more important $\eta K$ channel. It should be noted that the effect on the reconstruction of the phase is small but the reconstruction of the pole position might be affected. This has been shown in Ref. 45] for the $\kappa(800)$ and $\vec{P}=\overrightarrow{0}$.

Second, we quantify the effect if partial wave mixing is neglected (in the one-channel extraction scheme we are are using). In that case, Eq. (54) reduces to Eq. (55) which is the ordinary Lüscher equation for a boosted $S$-wave system. The results are shown with the dashdotted lines in Fig. 7. For very large $L$, we indeed observe that the extracted and the actual phase are similar. In other words, the $P$-wave decouples from the $S$-wave in the infinite volume limit as must be. However, even for $L>3 M_{\pi}^{-1}$ we already observe large deviations and for smaller box sizes than $L \sim 2 M_{\pi}^{-1}$, a reliable extraction of the phase, let alone the pole position of the $\kappa(800)$, is not possible any more.

In summary, it is crucial to take effects from partial 

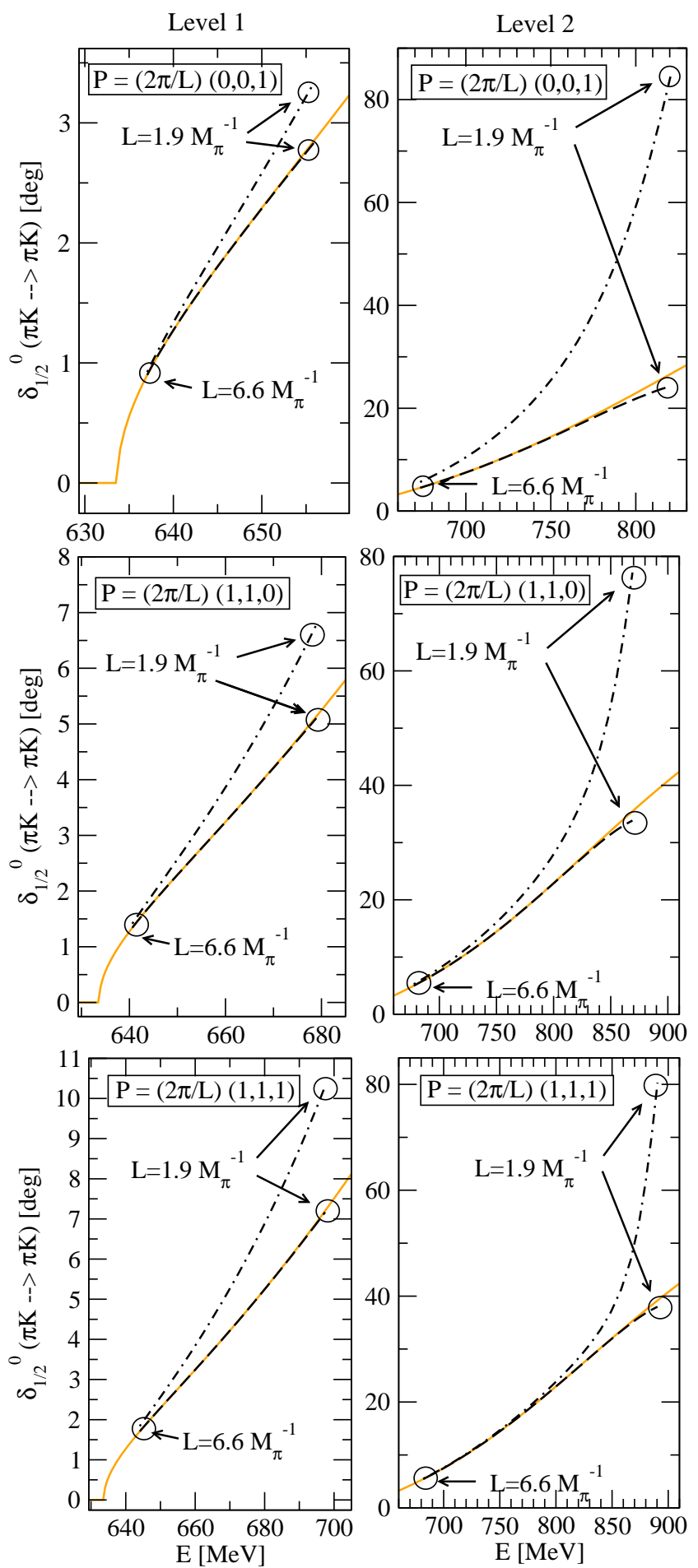

FIG. 7: Actual phase shifts [solid (orange) lines], identical to the result shown in Fig. 1 and the extraction from the first and second level, using Eq. (54) for the disentanglement (dashed lines) or Eq. (55) in which the partial wave mixing is neglected (dash-dotted lines). Results are shown for the first three boosts $\sim(0,0,1),(1,1,0)$, and $(1,1,1)$. In the figure, also the corresponding values of the box size $L$ are indicated.

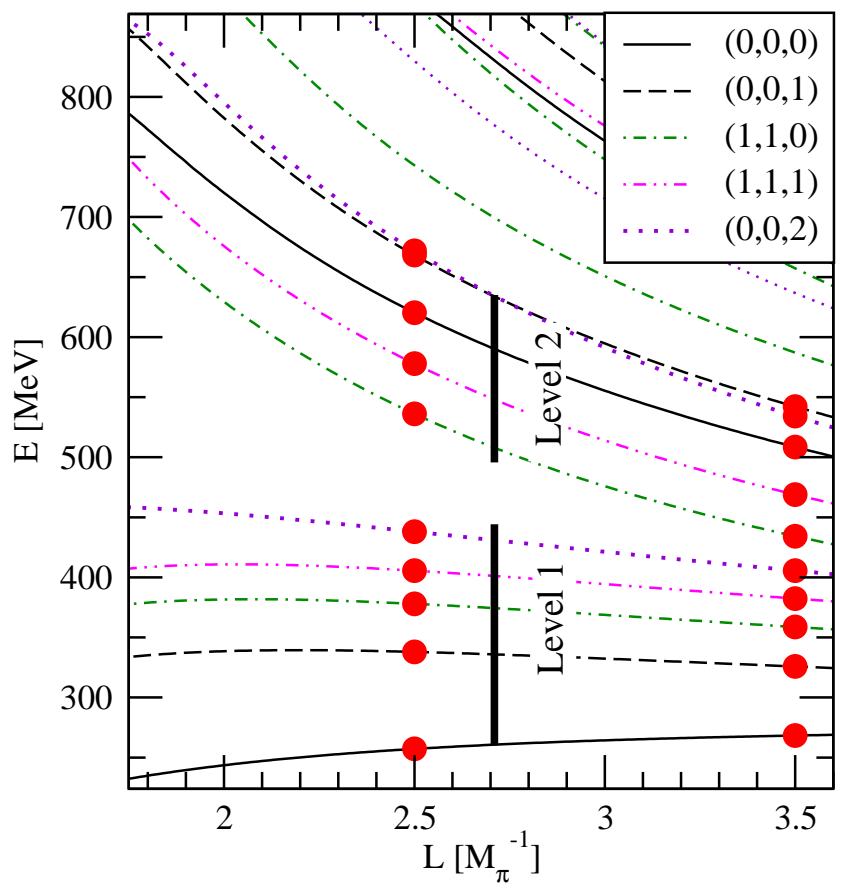

FIG. 8: Levels of the $(I=L=S=0)$ meson-meson system $\left[\sigma(600)\right.$ and $\left.f_{0}(980)\right]$ for different boosts. The symbols at $L=2.5 M_{\pi}^{-1}$ and $L=3.5 M_{\pi}^{-1}$ indicate the 20 synthetic data points taken for the reconstruction of phase and $\sigma(600)$ pole position [results in Figs. 10 and 11.

wave mixing into account while effects from inelastic channels can be neglected to a good accuracy as long as one considers energies only below the inelastic threshold. As a side remark, we would like to stress that in different physical contexts it might be crucial to use a two-channel extraction scheme, in particular if thresholds are close to each other as in case of the $\Lambda(1405)$ [37, 40, 49], or if one wants to extract phases and poles close to thresholds as in case of the $f_{0}(980)$ 32].

\section{THE $\sigma(600)$ IN A MOVING FRAME}

\section{A. Level spectrum and resonance extraction}

The first few levels for the $I=L=S=0$ quantum numbers are shown in Fig. 8 for the first five boosts. To obtain these levels, the $S$-wave two-channel potential $(\pi \pi, \bar{K} K)$ from the inverse amplitude method, $V_{S}^{(i j)} \equiv$ $V^{\mathrm{IAM}}$, given in Eq. (34), is used in Eq. (33) to determine the levels. For $V^{\mathrm{IAM}}$, the corresponding solution of Ref. [45] for the underlying hadronic interaction is used.

To quantify the expected error from partial wave mixing, we have calculated the level shift due to the $S$-wave mixing with the small isospin zero $D$-wave. Note that for the considered equal-mass case $(\pi \pi$ and $\bar{K} K)$, there is no $S P$-wave mixing even for $\vec{P} \neq 0$. Anyway, the $\pi \pi$ sys- 
tem in $P$-wave has isospin one. For the $S D$-wave mixing, the phenomenological parameterization of the $D$-wave of Ref. [79] is used that serves to construct a $D$-wave to $D$ wave transition $V_{D}^{(2,2)}$ as described in Appendix A. We do not include a $\bar{K} K$ channel in $D$-wave as there is no phenomenological reason for it and we are far below the $\bar{K} K$ threshold, anyway. We have, for the $A_{1}^{+}$representation, the levels given by Eq. (33) where

$$
\begin{aligned}
V & =\left(\begin{array}{ccc}
V_{S}^{(11)} & V_{S}^{(12)} & 0 \\
V_{S}^{(21)} & V_{S}^{(22)} & 0 \\
0 & 0 & V_{D}^{(22)}
\end{array}\right) \\
\tilde{G} & =\left(\begin{array}{ccc}
\tilde{G}_{00,00}^{R(1)} & 0 & 0 \\
0 & \tilde{G}_{00,00}^{R(2)} & \tilde{G}_{00,20}^{R(2)} \\
0 & \tilde{G}_{20,00}^{R(2)} & \tilde{G}_{20,20}^{R(2)}
\end{array}\right)
\end{aligned}
$$

for the boosts $\vec{P}=(2 \pi / L)(0,0,1),(2 \pi / L)(1,1,1)$, and $(2 \pi / L)(0,0,2)$. For the boost $\vec{P}=(2 \pi / L)(1,1,0)$,

$$
\begin{aligned}
V & =\left(\begin{array}{ccccc}
V_{S}^{(11)} & V_{S}^{(12)} & 0 & 0 & 0 \\
V_{S}^{(21)} & V_{S}^{(22)} & 0 & 0 & 0 \\
0 & 0 & V_{D}^{(22)} & 0 & 0 \\
0 & 0 & 0 & V_{D}^{(22)} & 0 \\
0 & 0 & 0 & 0 & V_{D}^{(22)}
\end{array}\right) \\
\tilde{G} & =\left(\begin{array}{ccccc}
\tilde{G}_{00,00}^{R(1)} & 0 & 0 & 0 & 0 \\
0 & \tilde{G}_{00,00}^{R(2)} & \tilde{G}_{00,2-2}^{R(2)} & \tilde{G}_{00,20}^{R(2)} & \tilde{G}_{00,22}^{R(2)} \\
0 & \tilde{G}_{2-2,00}^{R(2)} & \tilde{G}_{2-2,2-2}^{R(2)} & \tilde{G}_{2-2,20}^{R(2)} & \tilde{G}_{2-2,22}^{R(2)} \\
0 & \tilde{G}_{20,00}^{R(2)} & \tilde{G}_{20,2-2}^{R(2)} & \tilde{G}_{20,20}^{R(2)} & \tilde{G}_{20,22}^{R(2)} \\
0 & \tilde{G}_{22,00}^{R(2)} & \tilde{G}_{22,2-2}^{R(2)} & \tilde{G}_{22,20}^{R(2)} & \tilde{G}_{22,22}^{R(2)}
\end{array}\right)(64)
\end{aligned}
$$

where the index (1) labels the $\bar{K} K$ channel and (2) the $\pi \pi$ channel. The resulting $S D$-wave mixed levels of the $A_{1}^{+}$representation are shown with the (red) solid lines in Fig. 9] together with the $S$-wave levels without mixing from Fig. 8. As the figure shows, the level shift from mixing, $\Delta E$ can reach up to $10 \mathrm{MeV}$ at $L=2 M_{\pi}^{-1}$, but stays below $3 \mathrm{MeV}$ for $L=2.5 M_{\pi}^{-1}$ and larger. The shift $\Delta E$ increases not only for smaller $L$, but also for higher energies where $\delta_{D}$ is larger. Eq. (62) indeed shows that this is the expected behavior.

Note that the level shift from the $S D$-mixing is much smaller than the one of the $\pi K, \eta K$ system discussed in Sec. III because the $\pi \pi D$-wave is much smaller than the $\pi K P$-wave in which the $K^{*}(892)$ resides.

\section{B. Extraction of the $\sigma(600)$}

As the level shift from mixing is smaller than $3 \mathrm{MeV}$ for $L=2.5 M_{\pi}^{-1}$ and larger, we concentrate on these box sizes and can neglect the mixing; for the reconstruction of

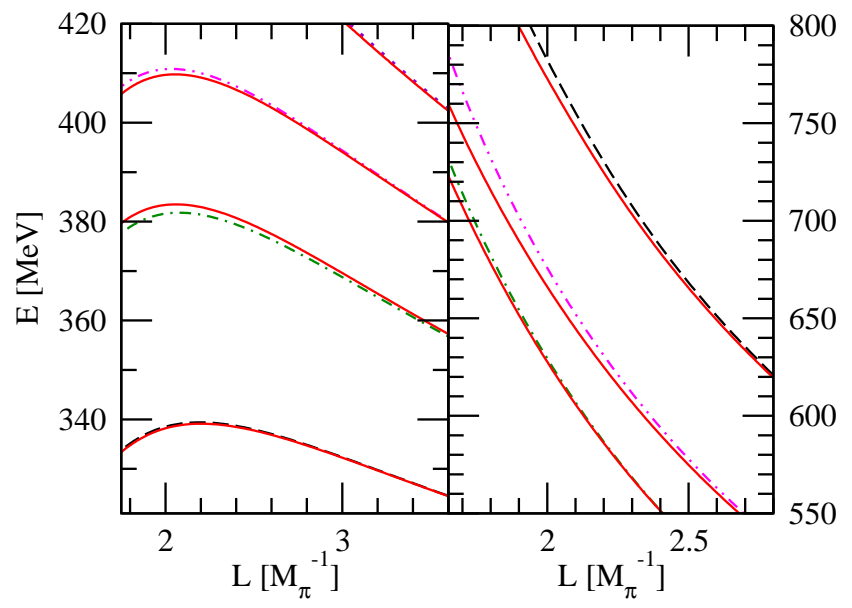

FIG. 9: Levels from the $A_{1}^{+}$representation as in Fig. 8 addition, the (red) solid lines show the levels mixed with $D$ wave.

the $\sigma(600)$ we will assume $10 \mathrm{MeV}$ errors on the synthetic data so that this assumption is safe. In this section, we concentrate on the extraction of the $\sigma(600)$ resonance and do not analyze the $f_{0}(980)$. The latter resonance would require a two-channel extraction scheme that has already been discussed in Ref. 32, and the extension to moving frames is in principle straightforward.

As Fig. 8 shows, with larger boosts the first and second level from the $A_{1}^{+}$representation move towards higher energies. In this way one can cover the entire energy region from threshold up to $E=700 \mathrm{MeV}$ with the boosted first two levels. This demonstrates the advantage lying in the use of moving frames: in the conventional Lüscher approach, $L$ is varied at $\vec{P}=\overrightarrow{0}$. This means that, first, many different lattice setups have to be calculated. Second, for the box sizes considered $2<L<3.6 M_{\pi}^{-1}$, the energy region around $E \sim 400-500 \mathrm{MeV}$, i.e. precisely where the real part of the pole position of the $\sigma(600)$ is located [80], is not covered by any level, making the extraction of the $\sigma(600)$ generically more difficult, as has also been noted in Ref. [45].

In the absence of actual lattice data, we generate 20 synthetic data points from the levels of Fig. 8 at two values of $L, L=2.5 M_{\pi}^{-1}$ and $L=3.5 M_{\pi}^{-1}$, as indicated with the dots in the figure. A $10 \mathrm{MeV}$ error is assigned to each data point. With such-defined data, the task is to reconstruct the $\pi \pi$ phase shifts and the $\sigma(600)$ pole.

For this, we use the parameterization of the onechannel potential from Ref. [45],

$$
\begin{aligned}
V^{\mathrm{fit}} & =\left(\frac{V_{2}-V_{4}^{\mathrm{fit}}}{V_{2}^{2}}\right)^{-1} \\
V_{4}^{\mathrm{fit}} & =a+b\left(s-s_{0}\right)+c\left(s-s_{0}\right)^{2}+d\left(s-s_{0}\right)^{3}
\end{aligned}
$$

with $V_{2} \equiv V_{\mathrm{LO}}$ the fixed LO term of the chiral expansion. In other words, we take the form of the inverse amplitude 
(c.f. Eq. (34)), leaving the LO term $V_{2}$ as given by chiral symmetry, and expand $V_{4}$ in powers of $s$. As expansion point we choose $s_{0}=(400 \mathrm{MeV})^{2}$.

The choice of this potential and its advantages have been extensively discussed in Ref. [45]. We denote here that the explicit inclusion of the model-independent, well-known lowest order term $V_{2}$ greatly helps stabilizing the extraction. The higher powers of $s$ account for corrections from the next-to-leading and higher order terms. Note that the polynomial NLO contributions [62] can be approximately taken account of, because the Mandelstam variable $t$ and $u$ can be expanded in $s$. The effect from the $\bar{K} K$ channel and its branch point at $E=2 M_{K}$, as well as the left-hand cut, is also absorbed in this expansion.

One could in principle include these non-analyticities explicitly in the fit potential. However, they are not well fixed because they lie much higher or much lower in energy. Given lattice data in a relatively narrow window in energy, no improvement is expected but instead large correlations of the corresponding new parameters will arise. The expansion of these effects in a power series in $s$, as provided in Eq. (65), allows for a systematic improvement and provides a set of parameters with relatively small correlations.

One should bear in mind, though, that this is an approximative procedure. Other than in Ref. [32], where the analytic form of the fit potential comprised the assumed hadronic interaction - this was possible because the model interaction was from lowest order only - the potential from Eq. (65) can only approximatively absorb the discussed effects. The strategy is then to perform different fits with increasing powers of $s$ until convergence is observed as discussed in Ref. [45] in detail.

\section{Extraction strategies with partial wave mixing}

For the $\pi \pi S$-wave sufficiently below the $\bar{K} K$ threshold, we can neglect the partial wave mixing as discussed before, but we give an outlook how to proceed in case it cannot be neglected as for the $\kappa / K^{*}$ system discussed in Sec. III On one hand, a separate extraction of $P$ - or $D$-waves is possible from the structure of the irreducible representations $B_{1}, B_{2}, E$ ( $P$-wave) and $A_{2}^{+}, B_{1}^{+}, B_{2}^{+}$, $E^{+}$( $D$-wave) as discussed following Eq. (55), see also Ref. [57] (we assume there is no mixing between $P$ - and $D$-wave). However, those phase shifts can only be extracted at scattering energies different from where the $A_{1}^{(+)}$levels are situated, from which the $S$-wave can be reconstructed via Eq. (54) in the one-channel case. For reference, see Figs. 3. to [5 It is then necessary to make some minimal assumptions on the $P$ - or $D$-wave phase shifts, such that the underlying potential can be expanded in energy as discussed in Sec. IVB, c.f. Eq. (65). This has also been recognized in Ref. [57].

Instead of determining first the higher partial wave and then the $S$-wave, it might be advantageous to simultane- ously fit the different levels with $V_{S}$ and $V_{P}$ (or $V_{S}$ and $V_{D}$ ), both of them expanded in energy as in Eq. (65). Such a procedure would be in analogy with the twochannel extraction scheme developed in Ref. 32] and is expected to be more efficient: the simultaneous fit of pure $P$-wave and $S P$-wave levels leads to smaller uncertainties in the infinite-volume limit than the two-step procedure discussed before.

A generalization to the multiple partial wave, multiple channel situation, using the corresponding matrices in channel- and partial wave-space as those of Eqs. (35, 36), is in principle straightforward but will require very high precision lattice data; see Ref. [32] where this issue is discussed for the two-channel case.

As performed in Sec. IVC for the $\sigma(600)$, there is no particular problem to generate pseudo-data for the $\kappa / K^{*}$-system and disentangle phase shifts following the strategy formulated here. However, to not overload this study, we concentrate on the somewhat simpler case of the $\sigma(600)$ in the next section.

\section{Results}

The analysis of the $\sigma(600)$ proceeds as described following Eq. (65) and in Ref. 45], with the fit potential $V^{\text {fit }}$ from Eq. (65). The fits are labeled according to the powers of $s$ used in $V^{\text {fit }}$. Once a minimum is found, parameter errors are determined. The parameter error for a parameter $a$ is defined by the range of $a$ in which $\chi^{2}<\chi_{\text {best }}^{2}+1$, under the constraint that all other parameters are optimized. Then, within the errors, random parameter sets are generated and only those sets kept for which $\chi^{2}<\chi_{\text {best }}^{2}+1$. For each of these sets, phase shift and pole position are calculated. The resulting bands and areas, for phase and pole position, respectively, are shown in Figs. 10 and 11. Note that the uncertainty area for the pole position in the $\left(s^{0}\right)$ fit, shown in Fig. 11, shrinks to a line. For the best $\chi^{2}$, pole positions are indicated with symbols in Fig. 11] The actual phase shift and pole position, derived from the hadronic interaction that was used to generate the synthetic data, are also indicated in the figures.

As visible for phase shifts and pole positions, fits with larger number of free parameters result (trivially) in larger uncertainties, and, of course, in a better $\chi^{2}$. For the central values of the pole positions, we observe that with an increasing number of parameters, the actual and the fitted pole positions get closer, but even the 4-parameter fit does not perfectly match the actual pole position although it has the best $\chi^{2}$ of all fits. As discussed in Sec. [1], there are terms of higher order in $s^{N}$, $N>3$, in the original potential that cause this small but finite discrepancy. In the fit of actual lattice data, one will have the same effect, of course.

As discussed in Ref. [45] the effect of the heavier channel - given by $\bar{K} K$ in the present case - usually can be well absorbed in the coefficients of the expansion of the 


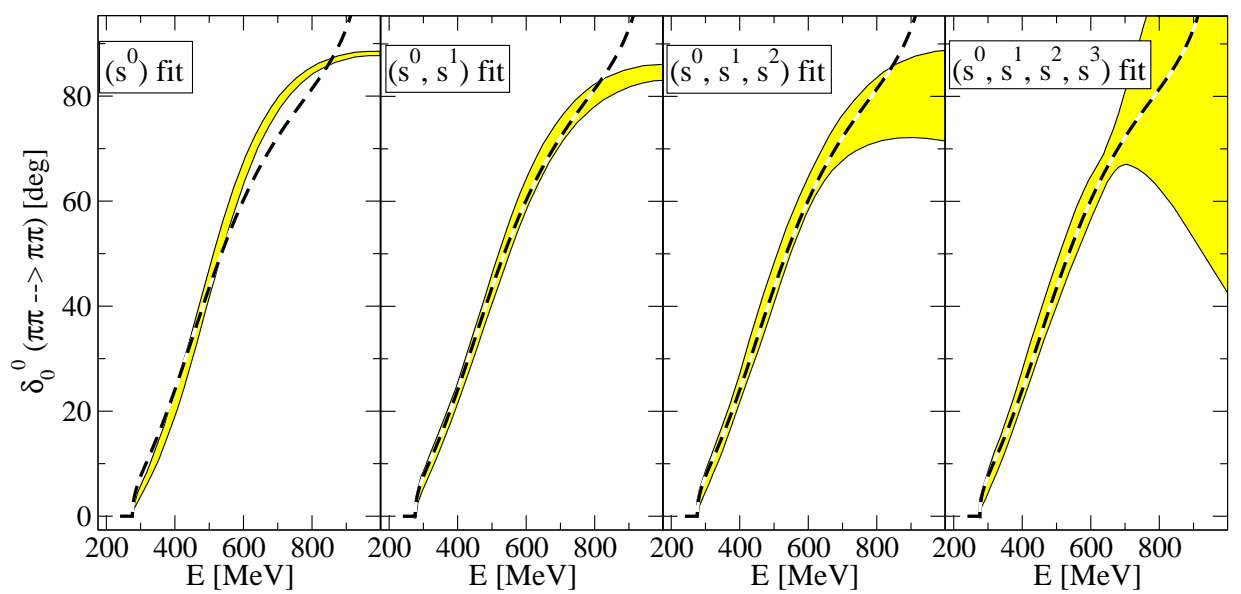

FIG. 10: Extracted phase shifts (bands) using synthetic data points as indicated in Fig. 8 with a $10 \mathrm{MeV}$ error for each data point. The actual phase shift is shown with the dashed lines.

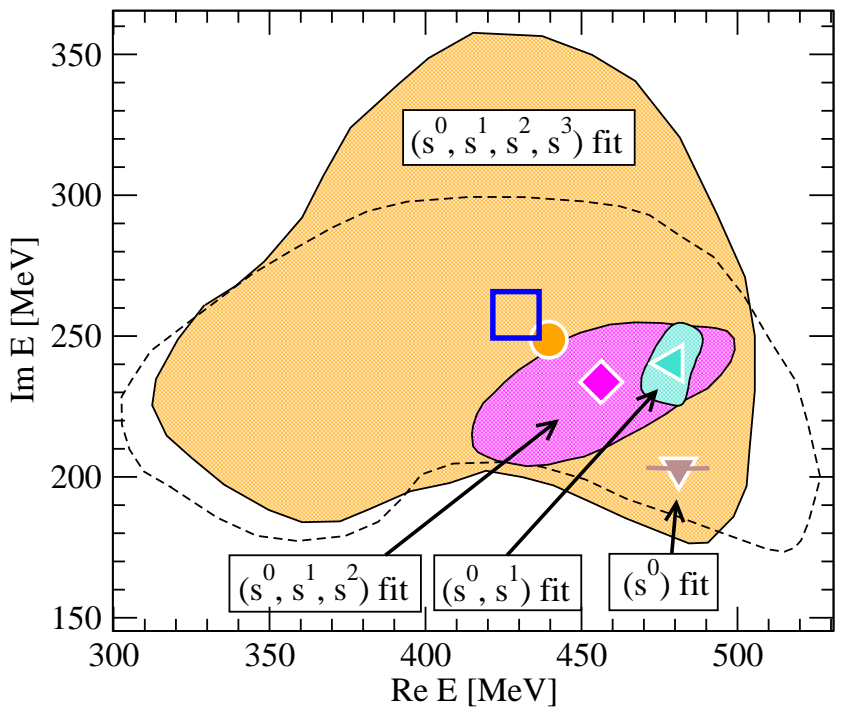

FIG. 11: Pole of the $\sigma(600)$ extracted from the synthetic data shown in Fig. 8. The actual pole position is indicated with the large square. Extracted pole positions for the different fits: triangle down: $\left(s^{0}\right)$ fit; triangle left: $\left(s^{0}, s^{1}\right)$ fit; diamond: $\left(s^{0}, s^{1}, s^{2}\right)$ fit; circle: $\left(s^{0}, s^{1}, s^{2}, s^{3}\right)$ fit. Also, uncertainties are shown (shaded areas). The dashed line shows the uncertainty from Ref. [45], where - instead of two volumes and five boosts as done here -6 different volumes had to be considered.

fit potential, but as the $\sigma(600)$ is very broad, small remaining discrepancies become large far in the complex plane. The same behavior was found in Ref. [45], where $L$ was varied to extract the $\sigma(600)$ pole. The situation cannot be improved by explicitly including the $\bar{K} K$ channel in the extraction process, in contrast to the case of the $f_{0}(980)$ where this is possible and necessary [32]. Here, i.e. still far below the $\bar{K} K$ threshold, the channel transitions $V_{\pi \pi \rightarrow \bar{K} K}$ and $V_{\bar{K} K \rightarrow \bar{K} K}$ are very weakly con- strained. The large number of new free parameters, tied to these additional transitions, would immediately lead to drastically increased uncertainties on the observables and large parameter correlations as has been tested.

We observe, in any case, that using synthetic data from values of $L$ smaller than $2.5 M_{\pi}^{-1}$, i.e. higher energies $E$, immediately helps to narrow down the uncertainties of the phase shifts at higher energies, shown in Fig. 10. However, in that case the central value of the pole position in the $\left(s^{0}, s^{1}, s^{2}, s^{3}\right)$ fit, shown with the circle in Fig. 11] starts to deviate considerably from the actual pole position (empty square), which is a sign of the increasing effect of the $\bar{K} K$ channel on the lattice data closest to the $\bar{K} K$ threshold. Choosing box sizes of $L=2.5 M_{\pi}^{-1}$ and $L=3.5 M_{\pi}^{-1}$ as done here provides, thus, a good compromise. These values of $L$, for which partial wave mixing can be safely neglected, is promising for the setup of an actual lattice simulation to extract the $\sigma(600)$.

Finally, we would like to compare the present results to those of Ref. 45] where - instead of two required volumes as is the case here -6 different volumes had to be considered, implying a much increased numerical effort for actual lattice calculations. The uncertainty of the $\sigma(600)$ pole position, coming from synthetic data with the same $10 \mathrm{MeV}$ error as used here, is shown with the dashed line in Fig. 11. The extension is of similar size as the one of the $\left(s^{0}, s^{1}, s^{2}, s^{3}\right)$ fit performed here.

Thus, with only two different volumes and using data from five boosts for each, one can expect results of similar precision than from six different values of $L$ without boost. This demonstrates that the proposed extraction method can be quite effective in the analysis of actual lattice data. 


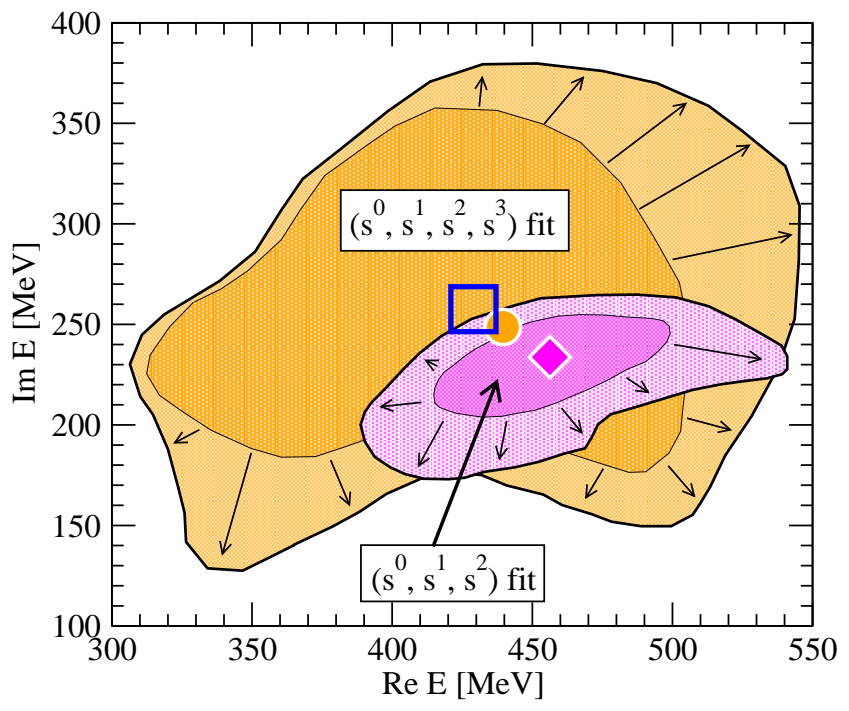

FIG. 12: Uncertainties of the $\sigma(600)$ pole position from the statistical uncertainty of the data. As indicated with the arrows, the uncertainties increase compared to those of Fig. [11] The cases of the $\left(s^{0}, s^{1}, s^{2}\right)$ and the $\left(s^{0}, s^{1}, s^{2}, s^{3}\right)$ fits are shown.

\section{Statistical error}

One should note that for the generation of the synthetic data we have so far only assigned an error, but not allowed the statistical fluctuation of the centroids of the error bars. In other words, we have assumed that the entire error is systematic. Here, we allow, in addition to the $10 \mathrm{MeV}$ error, a statistical fluctuation of the centroids by $5 \mathrm{MeV}$, as has been done in Ref. 32 for the extraction of the $f_{0}(980)$. The values of $10 \mathrm{MeV}$ for the error bar and $5 \mathrm{MeV}$ for its fluctuation are chosen to demonstrate the effect; when it comes to the analysis of actual lattice data, these values and in particular the fraction of the statistical in the total error have to be adapted, of course. Using the same procedure as in Ref. [32], we can estimate the resulting uncertainties, shown in Fig. 12 for two fits. As the figure shows, the uncertainties increase by around one third for the chosen values.

\section{SUMMARY}

The present study provides a formulation for the scattering of two particles confined in a finite box with total nonzero momentum, adapted to the chiral unitary framework. The idea is based on extending previously known techniques for zero momentum, discretizing the energy levels by imposing the boundary conditions in the moving frame.

Given a hadronic interaction, levels for the first five boosts $\vec{P}=(2 \pi / L)(0,0,0)$ to $(0,0,2)$ can be predicted and attributed to the subgroups of cubic symmetry. Em- ploying coupled-channel unitarized chiral perturbation theory including NLO terms, we derive the levels for the mixed-partial wave system with $I=1 / 2, S=-1$ and $L=0,1\left[\kappa(800)\right.$ and $K^{*}(892)$, respectively] as well as for the scalar sector with $I=0, S=0$ and $L=0,2$ where the $\sigma(600)$ resides.

We demonstrate for the $\kappa(800) / K^{*}(892)$ system that partial wave mixing is a very large effect for realistic box sizes and needs to be taken into account. To disentangle the $S$-wave from $P$ - or $D$-wave, we derive a set of equations in the one-channel formalism that are shown to be very precise as long as one stays below the inelastic thresholds.

Furthermore, we present a scheme in which the hadronic interaction is expanded in energy to allow for the extraction of the infinite volume limit, simultaneously fitting levels for different boosts and at different energies. The model-independent information from the lowest order in the chiral expansion is kept explicitly in this expansion, greatly stabilizing the fit to lattice data. Such statistical analyses can be used for actual lattice data, or, as done here, serve to determine promising lattice setups and the accuracy of lattice data to allow for reliable resonance extraction.

The method is tested for the example of the $\sigma(600)$. First, we show that for $L>2.5 M_{\pi}^{-1}$ effects from $S D$ wave mixing can be neglected. Second, synthetic latticedata are produced and analyzed. We find that with only two different box sizes one can expect a similar precision on the $\sigma(600)$ pole position as by varying as much as 6 different box sizes at zero total momentum.

Using information from moving frames is, thus, indeed rewarding since, with only a few different box sizes, phase shifts and resonance parameters of excited mesons can be determined.

\section{Acknowledgments}

We would like to thank Sasa Prelovsek and Luis Roca for useful discussions. This work is partly supported by DGICYT contracts FIS2006-03438, the Generalitat Valenciana in the program Prometeo and the EU Integrated Infrastructure Initiative Hadron Physics3 Project under Grant Agreement no. 283286. We also acknowledge the support by DFG (SFB/TR 16, "Subnuclear Structure of Matter"), by COSY FFE under contract 41821485 (COSY 106) and the Sino-German CRC 110 "Symmetries and the Emergence of Structure in QCD. A.R. acknowledges support of the Shota Rustaveli National Science Foundation (Project DI/13/02-100/11).

\section{Appendix A: Parameterization of the $D$-wave from Ref. [79]}

For convenience we quote the parameterization of the isospin zero $D$-wave from Ref. [79]. To construct the 
$\pi \pi D$-wave to $D$-wave transition $V_{D}^{(2,2)}$, we express it in terms of $\cot \delta_{D}$ given in Eq. (A9) of Ref. [79],

$$
\left[V_{D}^{(2,2)}\right]^{-1}=\operatorname{Re} G-\frac{p \cot \delta_{D}}{8 \pi E}
$$

valid up to the $\bar{K} K$ threshold $\left(E<2 M_{K}\right)$. Here,

$$
\begin{gathered}
\cot \delta_{D}=\frac{E}{2 p^{5}}\left(M_{f_{2}}^{2}-s\right) M_{\pi}^{2}\left[B_{0}+B_{1} w(s)\right], \\
w(s)=\frac{E-\sqrt{E_{0}^{2}-E^{2}}}{E+\sqrt{E_{0}^{2}-E^{2}}}, E_{0}=1050 \mathrm{MeV}
\end{gathered}
$$

where $M_{f_{2}}=1275.4 \mathrm{MeV}, B_{0}=12.40, B_{1}=10.06$, and $p$ is the relative momentum of the pions.
[1] Y. Nakahara, M. Asakawa and T. Hatsuda, Phys. Rev. D 60 (1999) 091503.

[2] K. Sasaki, S. Sasaki and T. Hatsuda, Phys. Lett. B 623 (2005) 208.

[3] N. Mathur, A. Alexandru, Y. Chen, S. J. Dong, T. Draper, I. Horvath, F. X. Lee and K. F. Liu et al., Phys. Rev. D 76 (2007) 114505.

[4] S. Basak, R. G. Edwards, G. T. Fleming, K. J. Juge, A. Lichtl, C. Morningstar, D. G. Richards and I. Sato et al., Phys. Rev. D 76 (2007) 074504.

[5] J. Bulava, R. G. Edwards, E. Engelson, B. Joo, H-W. Lin, C. Morningstar, D. G. Richards and S. J. Wallace, Phys. Rev. D 82 (2010) 014507.

[6] C. Morningstar, A. Bell, J. Bulava, E. Engelson, J. Foley, K. J. Juge, D. Lenkner and M. Peardon et al., AIP Conf. Proc. 1257 (2010) 779.

[7] J. Foley, J. Bulava, K. J. Juge, C. Morningstar, M. Peardon and C. H. Wong, AIP Conf. Proc. 1257 (2010) 789.

[8] M. G. Alford and R. L. Jaffe, Nucl. Phys. B 578 (2000) 367.

[9] T. Kunihiro et al. [SCALAR Collaboration], Phys. Rev. D 70 (2004) 034504.

[10] F. Okiharu, T. Doi, H. Ichie, H. Iida, N. Ishii, M. Oka, H. Suganuma and T. T. Takahashi, hep-ph/0507187.

[11] H. Suganuma, K. Tsumura, N. Ishii and F. Okiharu, PoS LAT 2005 (2006) 070.

[12] H. Suganuma, K. Tsumura, N. Ishii and F. Okiharu, Prog. Theor. Phys. Suppl. 168 (2007) 168.

[13] C. McNeile and C. Michael [UKQCD Collaboration], Phys. Rev. D 74 (2006) 014508.

[14] A. Hart et al. [UKQCD Collaboration], Phys. Rev. D 74 (2006) 114504.

[15] H. Wada, T. Kunihiro, S. Muroya, A. Nakamura, C. Nonaka and M. Sekiguchi, Phys. Lett. B 652 (2007) 250.

[16] S. Prelovsek, C. Dawson, T. Izubuchi, K. Orginos and A. Soni, Phys. Rev. D 70 (2004) 094503.

[17] S. Prelovsek, T. Draper, C. B. Lang, M. Limmer, K. F. Liu, N. Mathur and D. Mohler, Conf. Proc. C 0908171 (2009) 508.

[18] S. Prelovsek, T. Draper, C. B. Lang, M. Limmer, K.F. Liu, N. Mathur and D. Mohler, Phys. Rev. D 82 (2010) 094507.

[19] H. -W. Lin et al. [Hadron Spectrum Collaboration], Phys. Rev. D 79 (2009) 034502.

[20] C. Gattringer, C. Hagen, C. B. Lang, M. Limmer, D. Mohler and A. Schäfer, Phys. Rev. D 79 (2009)
054501.

[21] G. P. Engel et al. [BGR [Bern-Graz-Regensburg] Collaboration], Phys. Rev. D 82 (2010) 034505.

[22] M. S. Mahbub, W. Kamleh, D. B. Leinweber, A. O Cais and A. G. Williams, Phys. Lett. B 693 (2010) 351.

[23] B. J. Menadue, W. Kamleh, D. B. Leinweber and M. S. Mahbub, Phys. Rev. Lett. 108 (2012) 112001.

[24] R. G. Edwards, J. J. Dudek, D. G. Richards and S. J. Wallace, Phys. Rev. D 84 (2011) 074508.

[25] S. Prelovsek, C. B. Lang, D. Mohler and M. Vidmar, PoS LATTICE 2011 (2011) 137.

[26] Z. Fu, JHEP 1201, 017 (2012).

[27] C. B. Lang, L. Leskovec, D. Mohler and S. Prelovsek, arXiv:1207.3204 [hep-lat].

[28] V. Bernard, U.-G. Meißner and A. Rusetsky, Nucl. Phys. B 788 (2008) 1.

[29] V. Bernard, M. Lage, U.-G. Meißner and A. Rusetsky, JHEP 0808 (2008) 024.

[30] V. Bernard, D. Hoja, U.-G. Meißner and A. Rusetsky, JHEP 0906 (2009) 061.

[31] V. Bernard, M. Lage, U.-G. Meißner and A. Rusetsky, JHEP 1101 (2011) 019.

[32] M. Döring, U.-G. Meißner, E. Oset, A. Rusetsky, Eur. Phys. J. A 47 (2011) 131.

[33] M. Lüscher, Commun. Math. Phys. 105 (1986) 153.

[34] M. Lüscher, Nucl. Phys. B 354 (1991) 531.

[35] S. He, X. Feng and C. Liu, JHEP 0507 (2005) 011.

[36] C. Liu, X. Feng and S. He, Int. J. Mod. Phys. A 21 (2006) 847.

[37] M. Lage, U.-G. Meißner and A. Rusetsky, Phys. Lett. B 681 (2009) 439.

[38] K. Polejaeva and A. Rusetsky, arXiv:1203.1241 [hep-lat].

[39] S. Kreuzer and H. W. Grießhammer, Eur. Phys. J. A 48 (2012) 93.

[40] M. Döring, J. Haidenbauer, U.-G. Meißner and A. Rusetsky, Eur. Phys. J. A 47 (2011) 163.

[41] M. Döring, C. Hanhart, F. Huang, S. Krewald and U.G. Meißner, Phys. Lett. B 681 (2009) 26.

[42] M. Döring, C. Hanhart, F. Huang, S. Krewald and U.G. Meißner, Nucl. Phys. A 829 (2009) 170.

[43] M. Döring, C. Hanhart, F. Huang, S. Krewald, U.G. Meißner and D. Rönchen, Nucl. Phys. A 851 (2011) 58.

[44] A. Martinez Torres, L. R. Dai, C. Koren, D. Jido and E. Oset, Phys. Rev. D 85 (2012) 014027.

[45] M. Döring and U.-G. Meißner, JHEP 1201 (2012) 009. 
[46] L. Roca and E. Oset, Phys. Rev. D 85 (2012) 054507.

[47] J.-J. Xie and E. Oset, arXiv:1201.0149 [hep-ph].

[48] H. -X. Chen and E. Oset, arXiv:1202.2787 [hep-lat].

[49] A. Martinez Torres, M. Bayar, D. Jido and E. Oset, arXiv:1202.4297 [hep-lat].

[50] V. Bernard, D. Hoja, U.-G. Meißner and A. Rusetsky, arXiv:1205.4642 [hep-lat].

[51] K. Rummukainen and S. A. Gottlieb, Nucl. Phys. B 450 (1995) 397.

[52] C. h. Kim, C. T. Sachrajda and S. R. Sharpe, Nucl. Phys. B 727 (2005) 218.

[53] S. Bour, S. König, D. Lee, H.-W. Hammer and U.G. Meißner, Phys. Rev. D 84 (2011) 091503.

[54] S. R. Beane et al. [NPLQCD Collaboration], Phys. Rev. D 85 (2012) 034505.

[55] Z. Davoudi and M. J. Savage, Phys. Rev. D 84 (2011) 114502.

[56] Z. Fu, Phys. Rev. D 85 (2012) 014506.

[57] L. Leskovec and S. Prelovsek, arXiv:1202.2145 [hep-lat].

[58] J. J. Dudek, R. G. Edwards and C. E. Thomas, arXiv:1203.6041 [hep-ph].

[59] M. T. Hansen and S. R. Sharpe, arXiv:1204.0826 [heplat].

[60] R. A. Briceño and Z. Davoudi, arXiv:1204.1110 [hep-lat].

[61] J. A. Oller and U.-G. Meißner, Phys. Lett. B 500 (2001) 263.

[62] J. A. Oller, E. Oset and J. R. Pelaez, Phys. Rev. D 59 (1999) 074001 [Erratum-ibid. D 60 (1999) 099906] [Erratum-ibid. D 75 (2007) 099903].

[63] J. A. Oller and E. Oset, Nucl. Phys. A 620 (1997) 438 [Erratum-ibid. A 652 (1999) 407].

[64] M. Döring et al., to be submitted.

[65] J. Nieves and E. Ruiz Arriola, Phys. Rev. D 64 (2001) 116008.
[66] B. Borasoy, P. C. Bruns, U.-G. Meißner and R. Nißler, Eur. Phys. J. A 34 (2007) 161.

[67] P. C. Bruns, M. Mai and U.-G. Meißner, Phys. Lett. B 697 (2011) 254.

[68] A. Dobado and J. R. Pelaez, Phys. Rev. D 56, 3057 (1997).

[69] J. Nieves, M. Pavon Valderrama and E. Ruiz Arriola, Phys. Rev. D 65 (2002) 036002.

[70] A. Gomez Nicola and J. R. Pelaez, Phys. Rev. D 65 (2002) 054009.

[71] C. Hanhart, J. R. Pelaez and G. Rios, Phys. Rev. Lett. 100 (2008) 152001.

[72] J. Nebreda and J. R. Pelaez, Phys. Rev. D 81 (2010) 054035 .

[73] M. Göckeler, R. Horsley, M. Lage, U.-G. Meißner, P. E. L. Rakow, A. Rusetsky, G. Schierholz and J. M. Zanotti, arXiv:1206.4141 [hep-lat].

[74] T. Luu and M. J. Savage, Phys. Rev. D 83 (2011) 114508.

[75] D. Aston, N. Awaji, T. Bienz, F. Bird, J. D'Amore, W. M. Dunwoodie, R. Endorf and K. Fujii et al., Nucl. Phys. B 296 (1988) 493.

[76] J. A. Oller and E. Oset, Phys. Rev. D 60 (1999) 074023.

[77] R. Mercer, P. Antich, A. Callahan, C. Y. Chien, B. Cox, R. Carson, D. Denegri and L. Ettlinger et al., Nucl. Phys. 32B (1971) 381.

[78] P. Estabrooks, R. K. Carnegie, A. D. Martin, W. M. Dunwoodie, T. A. Lasinski and D. W. G. S. Leith, Nucl. Phys. B 133 (1978) 490.

[79] R. Garcia-Martin, R. Kaminski, J. R. Pelaez, J. Ruiz de Elvira and F. J. Yndurain, Phys. Rev. D 83 (2011) 074004.

[80] I. Caprini, G. Colangelo, J. Gasser, H. Leutwyler, Phys. Rev. D68, 074006 (2003). 